\title{
Lipin 2/3 phosphatidic acid phosphatases maintain phospholipid homeostasis to regulate chylomicron synthesis
}

\author{
Peixiang Zhang, ${ }^{1}$ Lauren S. Csaki, ${ }^{1}$ Emilio Ronquillo, ${ }^{1}$ Lynn J. Baufeld, ${ }^{1}$ Jason Y. Lin, ${ }^{1}$ Alexis Gutierrez, ${ }^{1}$ Jennifer R. Dwyer, ${ }^{1}$ \\ David N. Brindley, ${ }^{2}$ Loren G. Fong, ${ }^{3}$ Peter Tontonoz, ${ }^{4,5}$ Stephen G. Young, ${ }^{3,5}$ and Karen Reue ${ }^{1,5}$ \\ 'Department of Human Genetics, David Geffen School of Medicine at UCLA, Los Angeles, California, USA. ${ }^{2}$ Signal Transduction Research Group, Department of Biochemistry, Faculty of Medicine and \\ Dentistry, University of Alberta, Edmonton, Alberta, Canada. ${ }^{3}$ Department of Medicine, Division of Cardiology, and ${ }^{4}$ Department of Pathology and Laboratory Medicine, David Geffen School of Medicine at \\ UCLA, Los Angeles, California, USA. ${ }^{5}$ Molecular Biology Institute, UCLA, Los Angeles, California, USA.
}

\begin{abstract}
The lipin phosphatidic acid phosphatase (PAP) enzymes are required for triacylglycerol (TAC) synthesis from glycerol 3-phosphate in most mammalian tissues. The 3 lipin proteins (lipin 1, lipin 2, and lipin 3) each have PAP activity, but have distinct tissue distributions, with lipin 1 being the predominant PAP enzyme in many metabolic tissues. One exception is the small intestine, which is unique in expressing exclusively lipin 2 and lipin 3. TAG synthesis in small intestinal enterocytes utilizes 2-monoacylglycerol and does not require the PAP reaction, making the role of lipin proteins in enterocytes unclear. Enterocyte TAGs are stored transiently as cytosolic lipid droplets or incorporated into lipoproteins (chylomicrons) for secretion. We determined that lipin enzymes are critical for chylomicron biogenesis, through regulation of membrane phospholipid composition and association of apolipoprotein B48 with nascent chylomicron particles. Lipin 2/3 deficiency caused phosphatidic acid accumulation and mammalian target of rapamycin complex 1 (mTORC1) activation, which were associated with enhanced protein levels of a key phospholipid biosynthetic enzyme (CTP:phosphocholine cytidylyltransferase a) and altered membrane phospholipid composition. Impaired chylomicron synthesis in lipin 2/3 deficiency could be rescued by normalizing phospholipid synthesis levels. These data implicate lipin $2 / 3$ as a control point for enterocyte phospholipid homeostasis and chylomicron biogenesis.
\end{abstract}

\section{Introduction}

The small intestine absorbs more than $90 \%$ of dietary triacylglycerol (TAG), which is primarily hydrolyzed into 2-monoacylglycerol (2-MAG) and unesterified fatty acids in the intestinal lumen of nonruminant animals $(1,2)$. After absorption into enterocytes, the products of TAG digestion are reassembled into TAG, which may be transiently stored as cytosolic lipid droplets or incorporated into intestinal lipoproteins (chylomicrons) that are secreted into the lymphatics (1-6).

In most mammalian tissues, TAG is synthesized by the glycerol 3-phosphate pathway through the sequential addition of fatty acid moieties to a glycerol 3-phosphate backbone $(7,8)$. The phosphate group is removed by lipin phosphatidic acid (PA) phosphatase (PAP) enzymes to generate diacylglycerol (DAG), which is converted to TAG through the action of diacylglycerol acyltransferase (DGAT) enzymes or to zwitterionic phospholipids.

\section{Related Commentary: p. 58}

Conflict of interest: The authors have declared that no conflict of interest exists. License: Copyright 2019, American Society for Clinical Investigation.

Submitted: May 31, 2018; Accepted: October 9, 2018

Reference information: J Clin Invest. 2019;129(1):281-295.

https://doi.org/10.1172/JCl122595.
The glycerol 3-phosphate pathway is critical for TAG synthesis in adipose tissue, liver, skeletal muscle, and many other tissues $(9,10)$. In the intestine, however, the monoacylglycerol pathway is thought to be the predominant TAG-synthesis pathway. This pathway utilizes 2-MAG, the major product of fat digestion, as the substrate for TAG synthesis through the sequential action of monoacylglycerol acyltransferase (MGAT) and DGAT enzymes $(5,11)$. Studies of MGAT2-deficient mice support a critical role for this pathway in intestinal TAG synthesis (12). Nevertheless, more than half a century ago, investigators detected significant levels of $\mathrm{Mg}^{2+}$-dependent PAP activity that is specific to the glycerol 3-phosphate pathway in the intestinal mucosa from multiple animal species (13-16). They speculated that the 2-MAG pathway could be responsible for intestinal TAG synthesis, whereas PAP activity could have a role in phospholipid synthesis for mucosal cell proliferation. However, no direct evidence was offered to support this hypothesis, and the role of intestinal PAP activity has not been revisited since the identification of the lipin protein family.

The intestine and liver are unique in that newly synthesized TAG may be stored as cytosolic lipid droplets or may bud from the endoplasmic reticulum (ER) membrane toward the ER lumen, where it is assembled into chylomicrons (in enterocytes) or very low-density lipoproteins (VLDLs, in hepatocytes) (3). VLDL and chylomicron formation begins by cotranslational lipidation of 
A

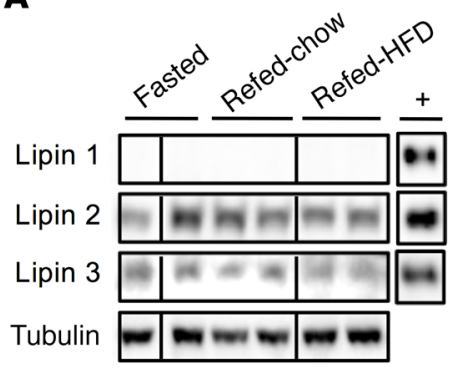

B

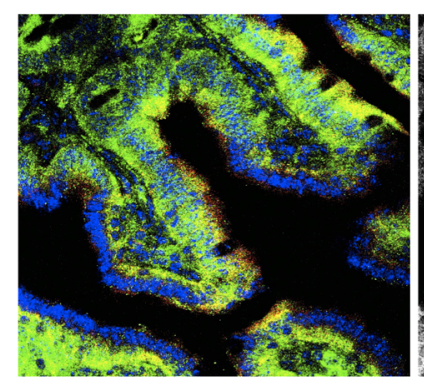

Lipin 2

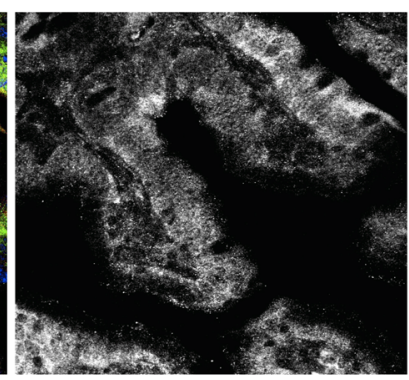

Lipin 3

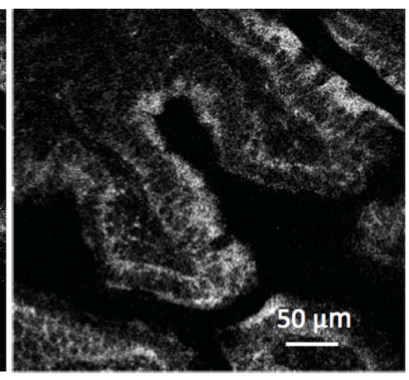

C

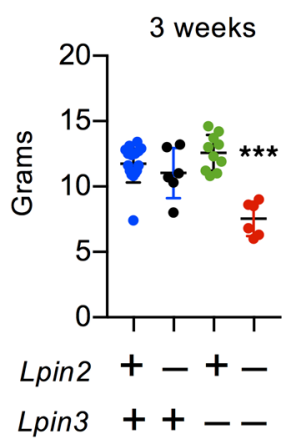

Body weight

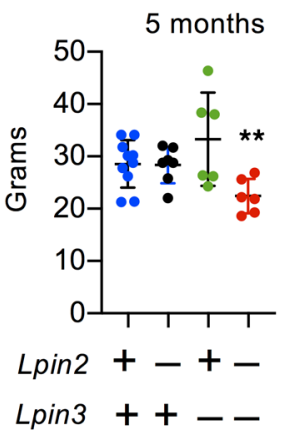

D

Intestinal
circumference
E

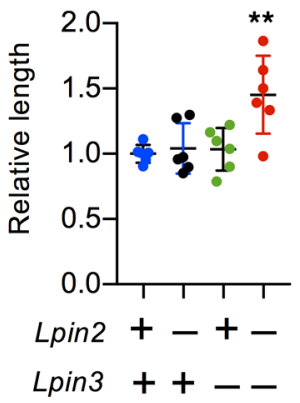

Intestinal length

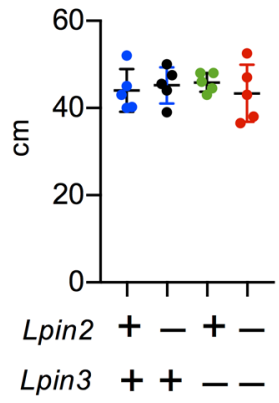

Weight change after 6 days HFD

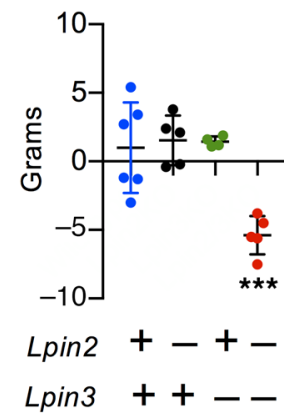

Figure 1. Lipin 2 and lipin 3 in mouse small intestine. (A) Immunoblot analysis of lipins 1, 2, and 3 in proximal small intestine (duodenum). Mice were fasted for 16 hours or were fasted 16 hours and refed 4 hours with a chow or high-fat diet (HFD), as indicated. Recombinant protein controls are shown in the right column (+). Lanes were all on the same blot, but noncontiguous, as indicated by vertical lines. This experiment is representative of 3 studies of lipin protein levels in small intestine from fasted mice, and 1 experiment in mice that were refed chow or HFD. (B) Localization of endogenous lipin 2 (green) and lipin 3 (red) in mouse duodenum shown by confocal fluorescence microscopy. Duodenum was collected from mice fasted for 5 hours. (C and D) Lpin2/3-KO mice (Lpin $2^{-/-} L$ pin $3^{-/-}$) have reduced body weight (shown for 3 weeks and 5 months of age), increased intestinal circumference, and normal intestinal length compared with WT, Lpin2-KO, or Lpin3-KO mice. Data shown are average \pm SD, $n=6-9 /$ group. (E) Body-weight change in mice fed HFD for 6 days. Average \pm SD, $n=4-6$ /genotype. ${ }^{* *} P<0.01$, ${ }^{* * *} P<0.001$ vs. other groups by ANOVA.

apolipoprotein B (apoB) (apoB100 in liver, apoB48 in intestine) to generate primordial lipoprotein particles, which undergo a second lipidation step, likely by fusing with apoB-free lipid droplets in the ER lumen (17-19). These steps require the action of microsomal TAG transfer protein. The primordial chylomicrons, consisting of a phospholipid shell surrounding a lipid core of TAG and cholesterol esters, bud from the ER and fuse with the Golgi, where they acquire additional protein components (e.g., apoA-IV) $(2,20)$. The mature chylomicron particles bud from the Golgi, fuse with the plasma membrane, and are released into the intercellular space. An estimated $70 \%-80 \%$ of TAG synthesis for chylomicron production in nonruminants occurs via the MGAT pathway $(21,22)$. In addition to TAG, chylomicron biogenesis requires appropriate levels of phospholipids, which coat the surface of the particle. Chylomicrons are thought to be assembled from newly synthesized TAG and preformed phospholipids (23). However, little is known about factors that regulate TAG and phospholipid incorporation into chylomicrons versus storage as cytosolic lipid droplets.

To enhance our understanding of the glycerol 3-phosphate pathway in intestinal lipid homeostasis, here we investigate the role of PAP enzymes. PAP activity is conferred by 3 lipin protein family members - lipin 1, lipin 2, and lipin 3. All 3 lipin proteins have PAP activity, but distinct tissue distributions (24). Lipin pro- teins also interact with DNA-bound transcription factors to coactivate or corepress gene expression, which does not necessarily require PAP activity (25-30). It has been challenging to establish the physiological roles of individual lipin proteins owing to the fact that most mammalian tissues express more than one of the lipin family members. Critical in vivo roles for lipin 1 have been most well established, and likely stem from its broad tissue distribution and highest PAP specific activity of the 3 lipin proteins (24, 31). Lipin 1 alone is responsible for PAP activity in skeletal muscle, and lipin 1 deficiency in both humans and mice leads to childhood rhabdomyolysis $(32,33)$. Lipin 1 is also responsible for most PAP activity in heart, and lipin 1 and lipin 3 together determine PAP activity in adipose tissue. Lipin 1 and lipin 2 are both active in lung and brain, and lipin 1, -2 , and -3 are active in liver, spleen, and kidney (34). Despite the presence of all 3 lipin proteins in liver, lipin 1 has a critical role in hepatic lipid homeostasis during the neonatal period. Lipin 1-deficient mice develop a fatty liver during the neonatal period and lasting for the first 2 weeks of life, but lipid accumulation is absent beyond 2 weeks of age (35). Specific roles for lipin 2 and lipin 3 have not been as clear as those for lipin 1 . Studies in mice lacking lipin 2 have impaired PAP activity in aging Purkinje cells, which becomes important for motor coordination as mice age (36). Humans with lipin 2 deficiency exhibit an auto- 
inflammatory condition associated with a role for lipin 2 in macrophage inflammasome activation $(37,38)$. Mice lacking lipin 3 are relatively normal, likely due to compensation by other lipin family members in most tissues (34).

Based on mRNA expression patterns, lipin 2 and lipin 3 are the most likely candidates to supply PAP activity in the small intestine $(9,31)$, but this has not been demonstrated experimentally. Here, we generated mice that lack both lipin 2 and lipin 3 and uncovered a critical role for these proteins in the regulation of intestinal lipid homeostasis and chylomicron production. Our findings reveal that lipin-mediated regulation of phospholipid synthesis in enterocytes is a critical control point for early stages of chylomicron assembly. Specifically, lipin 2/3 PAP activity regulates PA levels and mammalian target of rapamycin complex 1 (mTORC1) activation, which influences the protein levels of a key phospholipid biosynthetic enzyme (CTP:phosphocholine cytidylyltransferase $\alpha[\mathrm{CCT} \alpha]$ ) and membrane phospholipid composition. These findings establish a key role for lipin $2 / 3$ in the regulation of enterocyte phospholipid homeostasis that is distinct from a role in TAG synthesis.

\section{Results}

Lipin 2 and lipin 3 localize to intestinal enterocytes. To determine which lipin PAP family members are present in intestinal enterocytes, we examined protein levels by Western blot analysis. We detected lipins 2 and 3, but not lipin 1, in small intestinal mucosa (Figure 1A; see complete unedited blots in the supplemental material available online with this article; https://doi.org/10.1172/ JCI122595DS1). Nutritional status did not affect lipin levels, which were similar in mice that had been fasted or refed with a chow or high-fat diet. Lipins 2 and 3 were also present in a human intestinal cell line (Caco-2 cells). LPIN2 was expressed at highest levels in undifferentiated Caco-2 cells, while LPIN3 expression was induced during differentiation, slightly earlier than the apical sodium-dependent bile acid transporter, which is expressed in mature enterocytes (Supplemental Figure 1A) (39).

We assessed the localization of lipin 2 and 3 proteins in wild-type (WT) mouse duodenum by immunohistochemistry. In duodenum from fasted mice, lipins 2 and 3 appeared as puncta dispersed throughout the enterocyte cytoplasm; their distributions overlapped substantially, but each occurred independently as well (Figure 1B). The localization of lipins 2 and 3 changed in the fed state. In intestines harvested 2 hours after oil gavage, the 2 lipins colocalized (yellow puncta in Supplemental Figure 1B). In HEK293 cells, lipins 2 and 3 coimmunoprecipitated, consistent with the observed spatial colocalization of a proportion of these lipin proteins (Supplemental Figure 1C). Lipin 1 and lipin 2 proteins are known to heterodimerize without effects on PAP activity (40), and the function of lipin $1 / 2$ dimers or lipin $2 / 3$ dimers is unknown at present.

Lipin 2/3 deficiency reduces body weight on chow diet, and causes weight loss on high-fat diet. To investigate the role of lipins 2 and 3 in intestinal lipid homeostasis, we examined knockout mice that lack lipin 2 (Lpin2 -KO), lipin 3 (Lpin3-KO), or both (Lpin2/3-KO). Lpin2$\mathrm{KO}$ and Lpin3-KO mice were born at the expected frequencies and had a normal body weight, as previously reported $(34,36)$. Lpin2/3KO weanlings were observed at about one-quarter of the expected
Mendelian frequency $\left(\chi^{2}[1, n=349]=29.39, P<10^{-7}\right)$, indicating a high rate of lethality prior to weaning. Neonatal Lpin2/3-KO mice were not easily identifiable by appearance; however, soon after weaning, they had significantly reduced body weight and remained underweight as they aged (Figure 1C). Lpin2/3-KO mice had reduced fat mass, but normal liver and kidney size (Supplemental Figure 2A). The small intestine of these mice was of normal length but increased in circumference by $40 \%$ (Figure 1D). To determine whether a fat-rich diet could overcome the reduced body weight and fat mass in Lpin2/3-KO mice, 5-month-old mice were fed a high-fat/high-carbohydrate diet. Within 6 days, the mice lost $20 \%$ of their body weight and were hypoglycemic, necessitating termination of the study (Figure 1E, Supplemental Figure 2B).

The reduced body weight in Lpin 2/3-KO mice led us to examine food intake and nutrient absorption. In chow-fed mice, we found that both Lpin3-KO and Lpin2/3-KO mice had slightly reduced food intake (Supplemental Figure 3A). Additionally, Lpin3-KO and Lpin2/3-KO mice exhibited reduced daily fecal output, fecal TAG content, and plasma levels of the lipid-soluble vitamin D (Supplemental Figure 3, B-D). Since these phenotypes occurred to a similar extent in mice lacking only lipin 3 as in mice lacking both lipin 2 and 3 , they cannot account for the reduced adiposity and body weight observed specifically in Lpin2/3-KO mice. Lpin 2/3-KO mice were unique in having elevated fecal cholesterol levels, consistent with a reduction in dietary cholesterol absorption in Lpin2/3-KO mice (Supplemental Figure 3D). We were unable to perform studies of food intake and nutrient absorption in mice fed a high-fat diet due to the rapid deterioration of Lpin2/3-KO mice within a few days of eating the high-fat diet (see Figure $1 \mathrm{E}$ and Supplemental Figure $2 \mathrm{~B}$ ). However, we quantified plasma lipoprotein levels in mice fed both chow diet and those fed the high-fat diet for 6 days. On both diets, Lpin2/3-KO mice were unique among the 4 genotypes in having reduced total cholesterol and high-density lipoprotein (HDL) cholesterol levels (the latter constitutes the vast majority of cholesterol-carrying lipoproteins in the mouse) (Supplemental Figure 3, E and F). Lpin3- and Lpin2/3-KO mice had slightly elevated LDL cholesterol levels on the chow diet.

Loss of lipin 2 and/or lipin 3 substantially reduced $\mathrm{Mg}^{2+}$ dependent PAP activity in intestine (Supplemental Figure 4A). Since residual PAP activity was detected in Lpin2/3-KO intestinal lysates, we wondered whether a compensatory induction of lipin 1 might occur in lipin 2/3-deficient intestine. Although Lpin1 mRNA levels increased 2-fold, lipin 1 protein was undetectable by Western blot (Supplemental Figure 4, B and C). This makes it unlikely that lipin 1 accounts for the background PAP activity in Lpin 2/3-KO intestine. The residual PAP activity may represent nonspecific activity in intestinal tissue extracts that contributes to background in the PAP assay.

Impaired postprandial chylomicron production in Lpin 2/3-KO mice. Histological analysis of small intestine showed normal morphology in mice lacking only lipin 2 or lipin 3. However, Lpin2/3-KO mice had hyperplastic villi with lipid engorgement in the epithelium, even in the fasted state; their droplets were rich in neutral lipids and accumulated in the cytosol of enterocytes (Figure 2A). Biochemical analysis revealed an accumulation of TAG, with no significant change in cholesterol ester levels (Figure 2B). Membrane stacks also accumulated in Lpin2/3-KO enterocytes in both fasted and postprandial states (Figure 2C). 
A
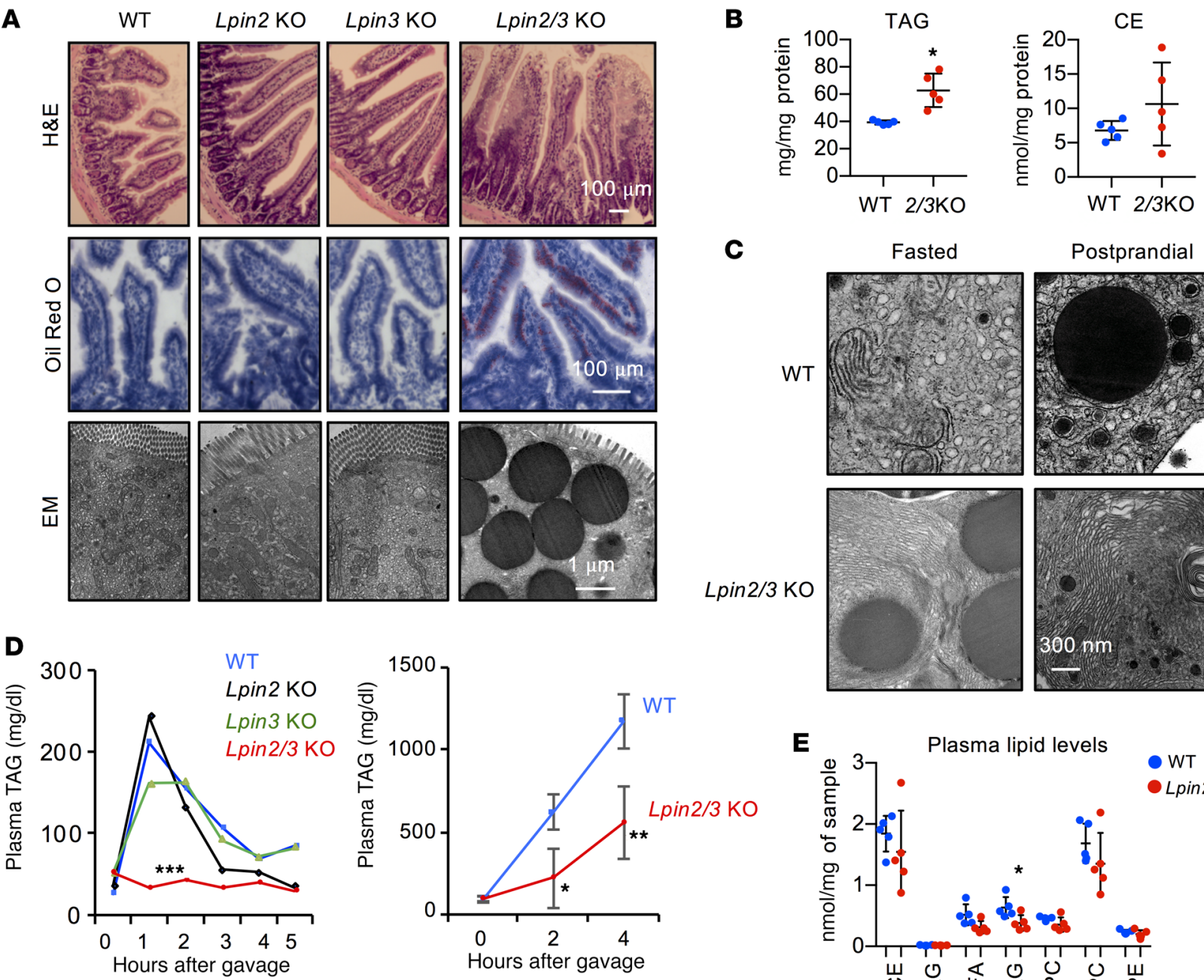

Postprandial

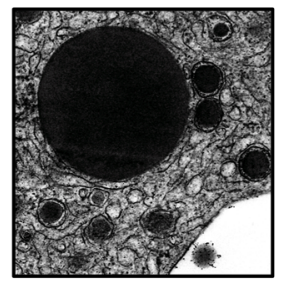

Lpin 2/3 KO
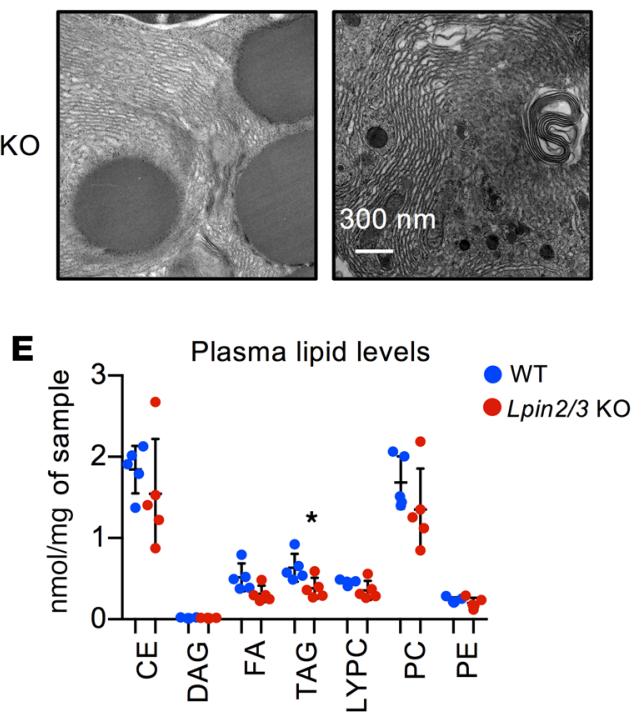

F
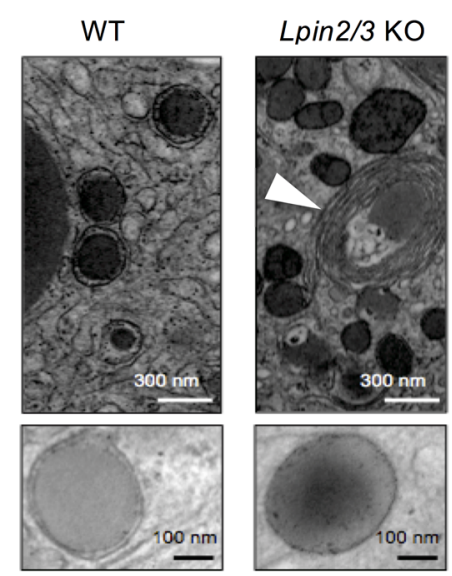

G

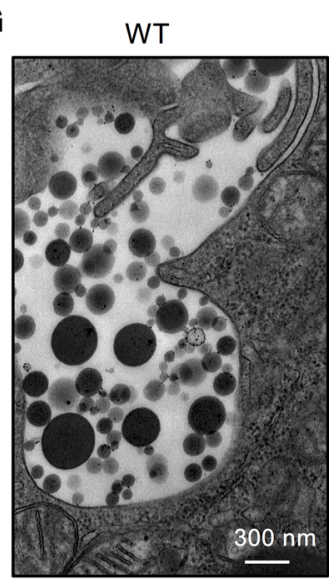

Lpin2/3 KO

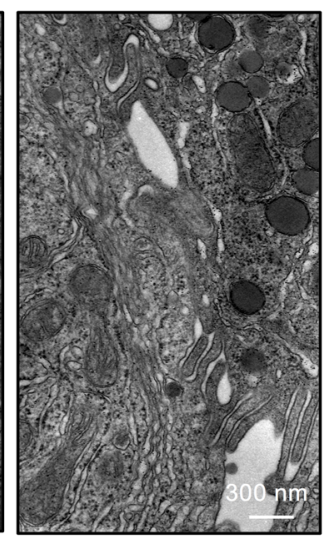

Lpin $2 / 3 \mathrm{KO}$

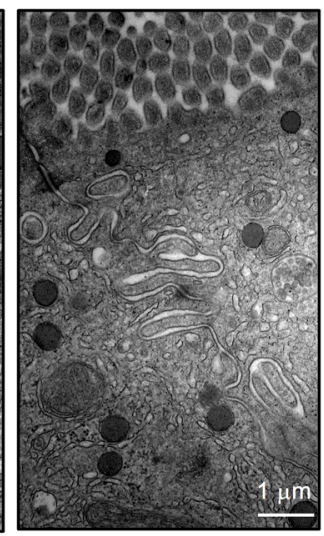


Figure 2. Impaired postprandial hyperlipidemia in Lpin2/3-KO mice. (A) Upper: Hyperplastic intestinal villi in Lpin2/3-KO mice (H\&E stain). Middle: Neutral lipid engorgement in intestinal epithelial cells in Lpin2/3-KO mice (oil red $\mathrm{O}$ stain). Lower: Electron micrographs show accumulation of cytosolic lipid droplets in enterocytes of fasted Lpin2/3-KO mice. Representative images from $n=2-3$ mice/genotype. (B) TAG and cholesterol ester (CE) concentrations in proximal intestine of WT and Lpin2/3-KO $(2 / 3 \mathrm{KO})$ mice. Data shown are average $\pm \mathrm{SD}, n=5$ /group. ${ }^{*} P<0.05$ vs. WT by $t$ test. FA, fatty acid; PC, phosphatidylcholine; LYPC, lyso-PC; PE, phosphatidylethanolamine. (C) Electron micrographs showing amplification of ER membranes in Lpin2/3-KO intestine, present in both fasted and postprandial states, and never detected in WT intestine. (D) Left: Analysis of postprandial hyperlipidemia in mice receiving oil gavage at time $0 ; n=$ 4-6/genotype. Right: Postprandial hyperlipidemia in mice pretreated with tyloxapol by tail vein injection before oil gavage. Average $\pm S D, n=3$ /group ${ }^{*} P<0.05$; ${ }^{* *} P<0.01$; ${ }^{* *} P<0.001$ vs. other groups by ANOVA. (E) Plasma lipids were quantified by electrospray ionization-tandem mass spectrometry in mice after a 5 -hour fast. Average $\pm \mathrm{SD}, n=5$ /genotype. ${ }^{*} P<0.05$ vs. WT by $t$ test. (F) Electron micrographs of proximal small intestine showing ER luminal lipid droplets or prechylomicron particles in WT mice and lipid droplets exclusively in the cytoplasm in Lpin2/3-KO mice. Note empty membrane stacks in Lpin2/3-KO mice (white arrowhead). Representative images from 3 mice/genotype. (C) Electron micrographs showing release of mature chylomicrons into the intercellular space in WT mice 2 hours after oil gavage. Junctions between Lpin2/3-KO cells remain intact with no evidence of chylomicron release. Representative electron micrographs from 3 mice/genotype.

The lipid accumulation in Lpin2/3-KO enterocytes suggested impaired secretion of intestinal chylomicrons. To assess this possibility, we examined plasma TAG levels after a bolus of olive oil by gastric gavage. As expected, plasma TAG levels increased transiently and rapidly returned to baseline levels in WT, Lpin2-KO, and Lpin3-KO mice (Figure 2D). In contrast, plasma TAG levels did not increase in Lpin 2/3-KO mice, suggesting severely impaired chylomicron production or secretion. To rule out rapid clearance of postprandial TAGs, an intragastric oil bolus was given to mice in which lipolytic processing of chylomicrons had been inhibited with tyloxapol. These studies confirmed the lower postprandial TAG levels in Lpin2/3-KO mice (Figure 2D), implying a defect in chylomicron production. Analysis of circulating lipids revealed reduced TAG levels in Lpin2/3-KO mice, but normal levels of cholesterol esters and major phospholipid species (Figure 2E).

Electron microscopy images of intestine 2 hours after oil gavage revealed prechylomicron particles in the ER lumen in WT intestinal cells; however, in Lpin2/3-KO cells, the lipid droplets were cytosolic, sometimes occurring near empty membrane stacks (Figure 2F). Furthermore, the release of mature chylomicron particles into the interstitial spaces at the basolateral surface of enterocytes was evident in WT but not Lpin2/3KO mice (Figure $2 \mathrm{G}$ ).

We assessed the levels of key chylomicron protein components. Levels of the major chylomicron protein, apoB48, were elevated in Lpin2/3-KO intestine (A), despite diminished mRNA levels (Supplemental Figure 5). Intestinal levels of additional chylomicron proteins, apoA-I and apoA-IV, were also increased by lipin 2/3 deficiency (Figure 3A). Levels of the lipid droplet protein perilipin 2 and the ER protein calnexin were dramatically elevated in Lpin2/3-KO intestine (Figure 3A), consistent with the accumulation of cytosolic lipid droplets and membrane stacks, respectively (see Figure 2). Gene-expression levels in Lpin2/3-KO intestine were normal for several factors that participate in chylomicron synthesis and intestinal lipid metabolism (e.g., microsomal TAG transfer protein, secretion-associated Ras-related GTPase $1 B$, the fatty acid transporter CD36, fatty acid binding proteins FABP1 and FABP2, TAG synthesis enzymes DGAT1 and DGAT2, and the TAG hydrolase ATGL; Supplemental Figure 5). The mRNA levels for Mogat2 (encoding MGAT2) were reduced in Lpin2/3-KO mice, perhaps in response to the accumulation of excess TAG within enterocytes.

Lipin PAP activity could potentially influence the levels of several cellular lipids, including the PAP substrate PA and immediate downstream product DAG, as well as phospholipids synthesized from DAG, such as phosphatidylcholine (PC). Intestinal lipid profiles revealed normal DAG levels in Lpin2/3-KO mice (Figure 3B), which are likely maintained through MGAT activity. PA levels were elevated in Lpin2/3-KO mice fed the high-fat diet (Figure $3 \mathrm{C}$ and Supplemental Figure 6A). Total PC levels were elevated in Lpin2/3-KO mice on chow and further elevated by a short-term high-fat diet (Figure 3D). Of note, PC composition in the intestine was altered in Lpin2/3-KO mice fed a chow or high-fat diet, with a reduced proportion of arachidonoyl-PC species (36:4 and 38:4 $\mathrm{PC}$ ) in Lpin2/3-KO mice (Figure 3E and Supplemental Figure 6B).

Consistent with the enhanced PC content in Lpin $2 / 3-\mathrm{KO}$ intestine, levels of CCT $\alpha$, the rate-limiting enzyme in PC synthesis, were substantially increased (Figure $3 \mathrm{~A}$ ). This was independent of CCT $\alpha$ mRNA (Pcyt) levels (Figure 3F), suggesting posttranscriptional regulation. CCT $\alpha$ protein levels are modulated posttranscriptionally by mTORC1, a major regulator of cellular anabolic processes such as protein and lipid synthesis (41). We therefore tested whether mTORC1 activity is enhanced in lipin 2/3-deficient intestine. mTORC1 was strongly activated in Lpin2/3-KO intestine, as indicated by increased phosphorylation (activation) of the mTORC1 target, p70s6 kinase (Figure 3G).

The association of elevated CCT $\alpha$ levels with impaired chylomicron production and lipid accumulation in Lpin2/3-KO intestine raised the question of whether a similar elevation in CCT $\alpha$ underlies the hepatic lipid accumulation that is a hallmark of lipin 1-deficient fatty liver dystrophy $(f l d)$ mice during the neonatal period $(35,42)$. We found that neither neonatal nor adult liver from lipin 1-deficient mice has elevated CCT $\alpha$ levels (Supplemental Figure 4D), suggesting a distinct mechanism for the lipid accumulation from that occurring in Lpin2/3-KO intestine.

Lipin 2 or 3 PAP activity is required for lipid compartmentalization in enterocytes. To investigate how lipins 2 and 3 influence chylomicron production, we visualized the fate of dietary lipids in Lpin2/3-KO intestine. BODIPY-labeled fatty acids and olive oil were given by gastric gavage, and the location of the BODIPY label was determined by fluorescence microscopy 2 hours later (Figure 4A). Fluorescent fatty acids were incorporated into abundant droplets throughout the apical and basolateral regions in WT enterocytes but accumulated in smaller lipid droplets distributed primarily in the apical region in Lpin2/3-KO enterocytes. Note that this technique allows detection only of the lipids provided in the oil gavage, and does not reveal the distribution of lipids that have previously accumulated within the Lpin2/3-KO enterocyte.

We assessed the localization of BODIPY-labeled lipids, relative to the ER markers calnexin and apoB, by confocal microscopy. In WT 
A

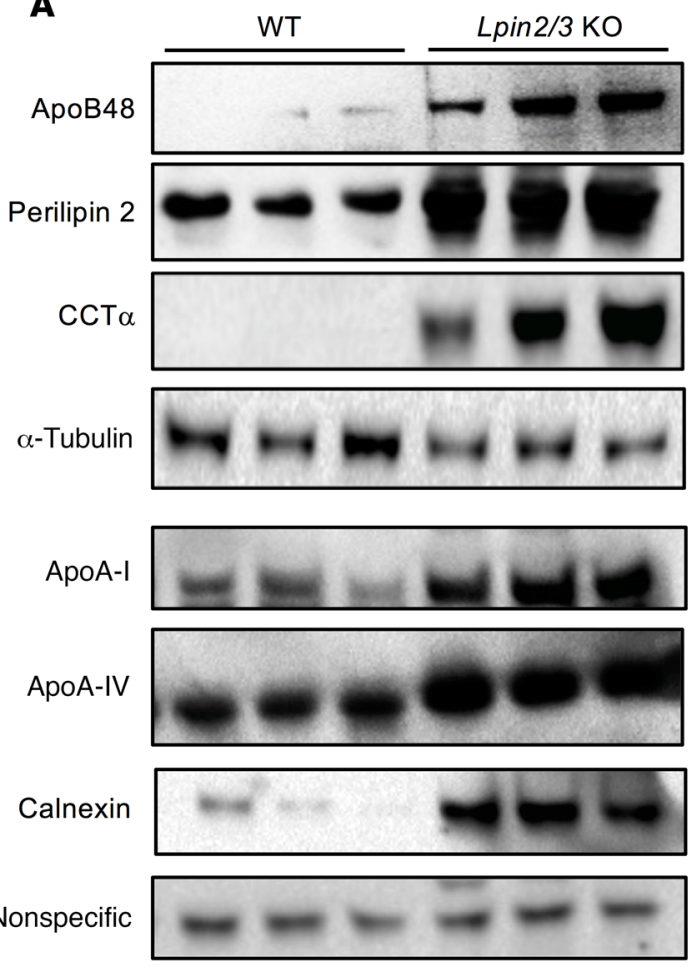

$\mathbf{F}$

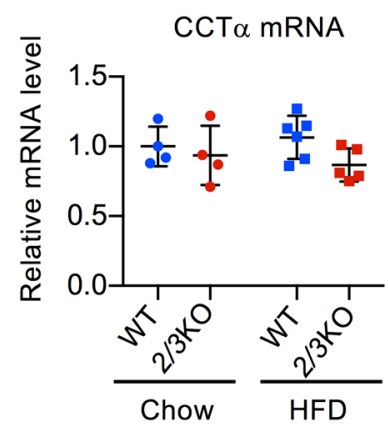

B

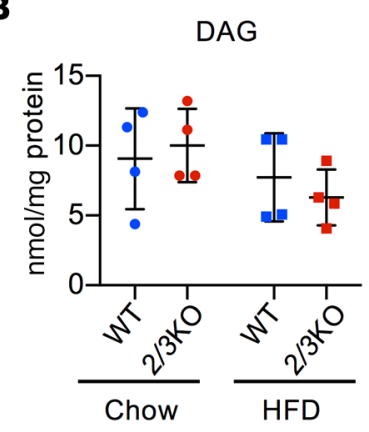

D

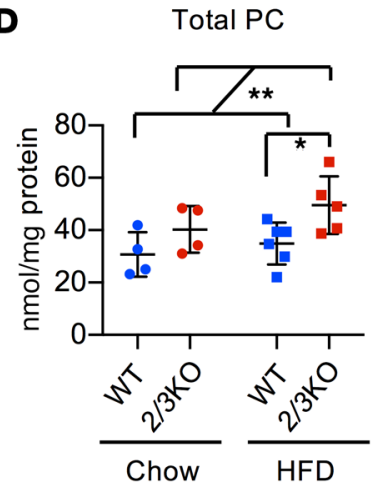

C

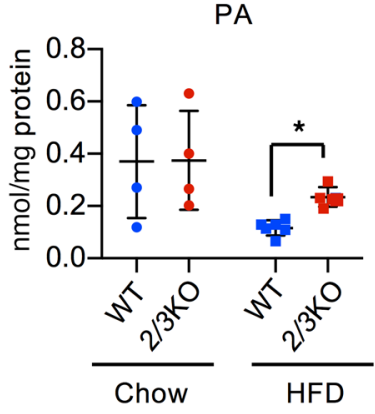

E

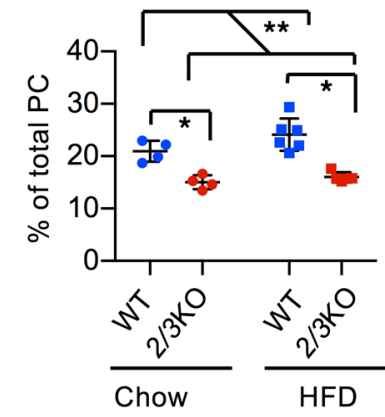

G

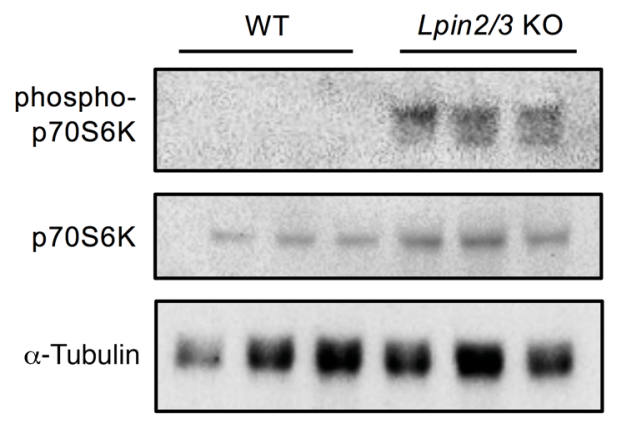

Figure 3. Increased levels of phospholipids and chylomicron-associated proteins in Lpin2/3-KO intestine. (A) Immunoblot analysis of intestinal protein levels for apolipoproteins apoB48, apoA-I, and apoA-IV, lipid droplet protein perilipin 2, phospholipid synthetic enzyme CCT $\alpha$, and ER protein calnexin. Mice were fed a high-fat diet for 6 days. WT, wild-type. All panels are the same protein samples. The top 4 blots were run contemporaneously, with $\alpha$-tubulin as a normalization control. The lower 4 blots were run contemporaneously with a nonspecific protein band as a normalizing control. Three biological replicates of each genotype are shown, and are representative of 5-8 samples of each genotype. (B-D) Proximal intestinal lipidomics analysis by electrospray ionizationtandem mass spectrometry in mice maintained on chow diet or fed a high-fat diet (HFD) for 6 days. Average \pm SD, $n=4-6$. (E) Altered PC composition in Lpin2/3-KO intestine, with reduced proportion of arachidonyl-PC species compared with WT. Average \pm SD, $n=4-6$. (F) CCT $\alpha$ (Pcyt) mRNA levels in intestine from mice indicated. Average \pm SD, $n=4-6$. (C) Immunoblot analysis of the mTORC1 target, p70S6 kinase, in intestine. Total and phosphorylated p70S6 kinase (Thr 389) were detected by specific antibodies. Blots were run contemporaneously with the same protein samples. ${ }^{*} P<0.05 ;{ }^{* *} P<0.01$ by ANOVA

enterocytes, calnexin was present near BODIPY-labeled lipids (Figure 4B). Calnexin staining and BODIPY-labeled lipid droplets were visible but did not colocalize in Lpin2/3-KO enterocytes. Furthermore, apoB was juxtaposed with lipid droplets in WT enterocytes, but not in Lpin2/3-KO mice (Figure 4C). In WT enterocytes, lipin 2 and apoB partially colocalized (Figure 4D), suggesting that lipin 2 is present at sites of lipid-apoB48 association during chylomicron assembly.

To further investigate how lipins 2 and 3 influence lipid compartmentalization and chylomicron biogenesis, we inactivated LPIN2 and LPIN3 in HT-29 human intestinal epithelial cells with
CRISPR/Cas9 genome editing (Supplemental Figure 7A). The absence of lipins 2 and 3 was confirmed by Western blotting (Supplemental Figure 7B). LPIN2/3-KO HT-29 cells had the same lipid compartmentalization defect seen in vivo (Figure 5A). LPIN2/3-KO cells formed a uniform population of lipid droplets, whereas WT cells synthesized droplets with a bimodal size distribution (Figure 5A). Total cellular TAG levels were similar in WT and LPIN2/3-KO HT-29 cells under basal culture conditions and after oleate loading (Figure 5B). However, PC levels were elevated in LPIN2/3-KO cells. Thus, it appears that lipins 2 and 3 are not required for the initial 
A
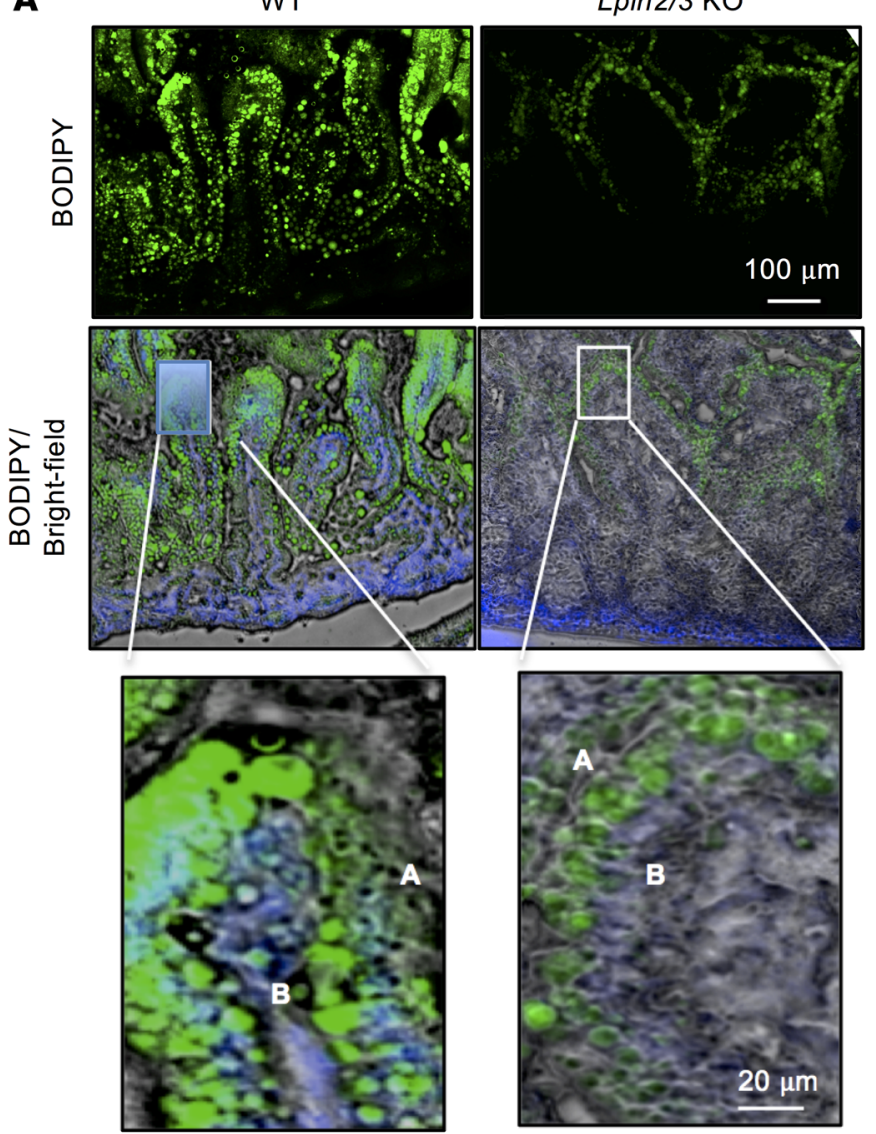

B
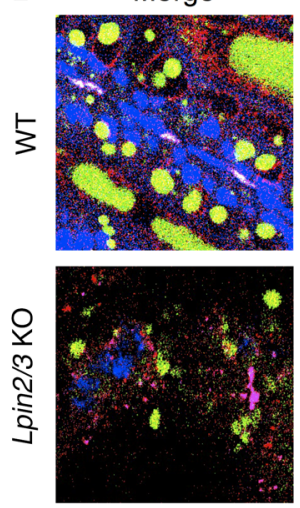

C
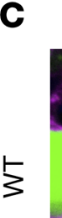

0
o
à
à
a
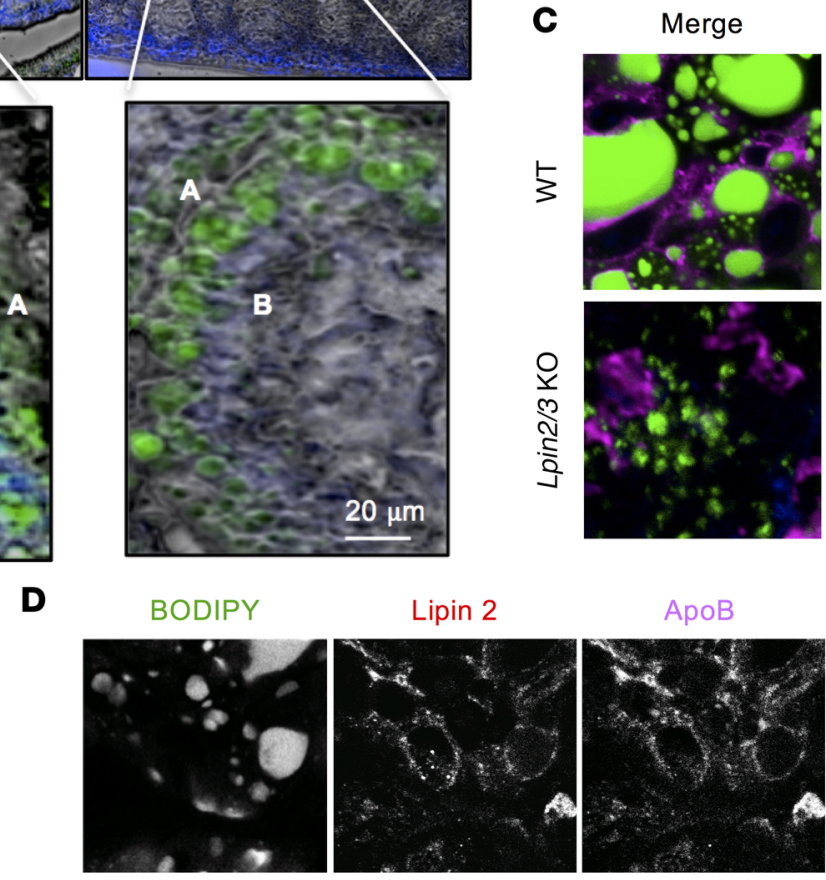

Lipin 2

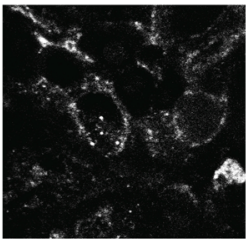

ApoB

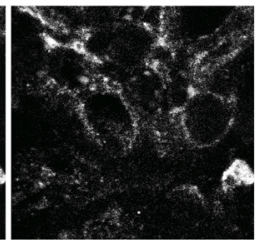

BODIPY
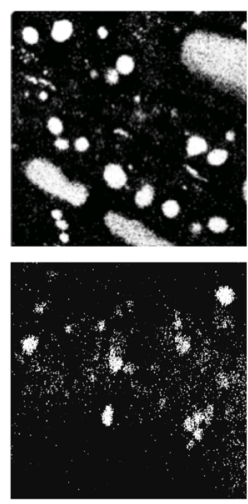

BODIPY
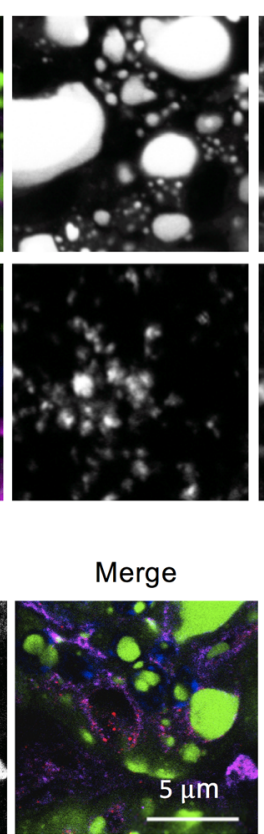

Calnexin

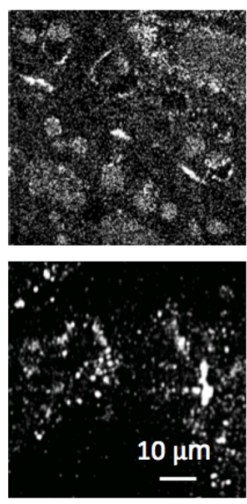

ApoB

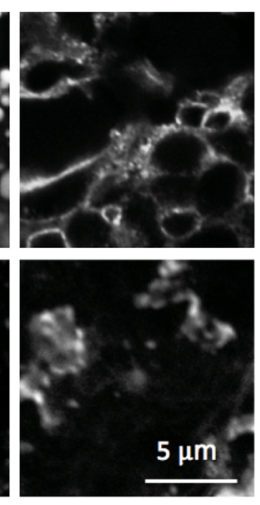

Figure 4. Aberrant lipid compartmentalization in response to an acute fat load in Lpin2/3-KO intestine. (A) Distribution of fluorescence in proximal small intestine of mice 2 hours after oral gavage of oil containing BODIPY-labeled fatty acids. Upper: Fluorescence image of lipid droplet (LD) distribution. Middle: Overlay of fluorescence image and bright field with DAPI nuclear stain (blue). Lower: Enlarged image of single villus tip showing LD localization to both apical (A) and basolateral (B) regions of enterocytes in WT enterocytes (left) and primarily to the apical region in Lpin2/3-KO enterocytes (right). Representative of 2 experiments. (B) LDs marked by BODIPY (green) associate with the ER protein calnexin in the proximal small intestine of WT but not Lpin2/3-KO mice. The image was taken 2 hours after oil gavage with BODIPY-labeled fatty acids. Nuclei are stained blue with DAPI. (C) ApoB associates with LDs in WT but not in Lpin2/3-KO enterocytes. Image taken 2 hours after oil gavage with BODIPY-labeled fatty acids. (D) Partial colocalization of lipin 2 and apoB proteins on the surface of LDs in the proximal small intestine of WT mice. The image was taken 2 hours after oil gavage with BODIPY-labeled fatty acids.

incorporation of fatty acids into TAG but are required for PC homeostasis and normal lipid droplet distribution.

Next, we determined whether TAG compartmentalization in enterocytes requires the PAP activity or coactivator function of lipins. LPIN2/3-KO HT-29 cells were reconstituted with WT lipin 2 (PAP and coactivator function) or with lipin $2^{\mathrm{D} 686 \mathrm{E}}$, a mutant that has only coactivator function (30). Only WT lipin 2 restored the bimodal lipid droplet distribution (Figure 5C) and reduced PC concentration (Figure 5D). Consistent with impaired PAP enzymatic activity, PA accumulated in Lpin2/3-KO intestine (Figure 3C and Supplemental
Figure 4A) and LPIN2/3-KO HT-29 cells, and PA levels were normalized when LPIN2/3-KO cells were reconstituted with WT lipin 2 (Figure 5E). These findings indicate that lipin PAP enzymatic activity is critical for normal TAG compartmentalization in enterocytes and raise the possibility that PC and PA levels influence this process.

Lipin PAP activity is required for assembly of apoB48 into lipidcontaining particles. We hypothesized that lipins 2 and 3 act during the association of TAG with apoB48 in the ER lumen, a crucial step of chylomicron production. To assess lipidation of apoB48, we fractionated WT and LPIN2/3-KO HT-29 cells by sucrose gradient 
A
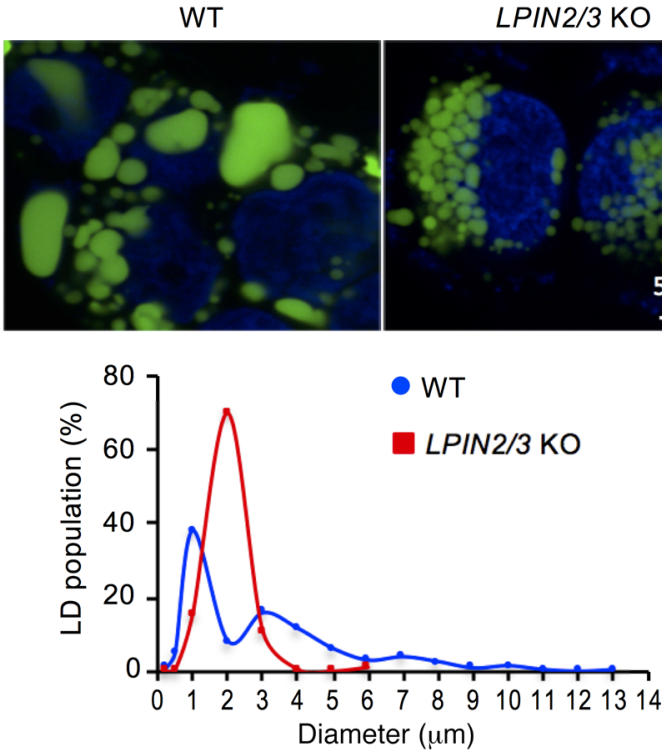

C
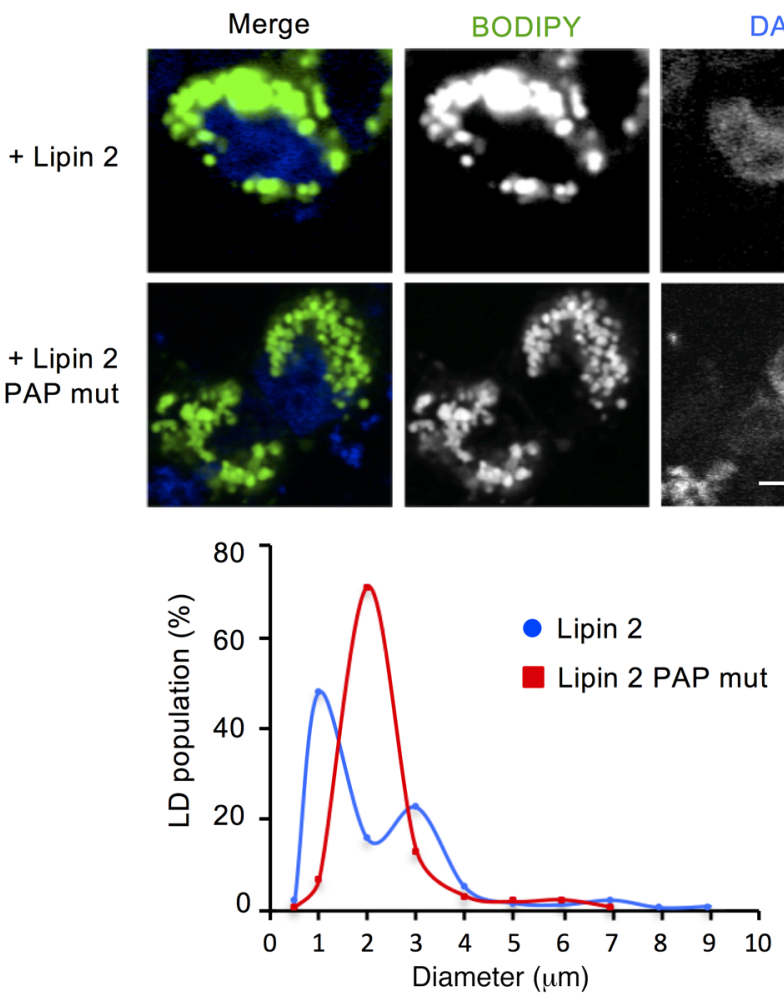

B

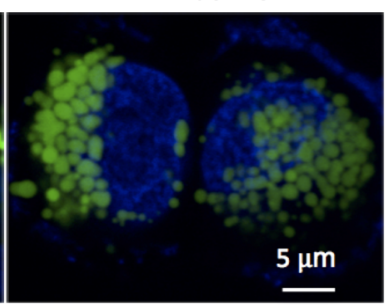

LPIN2/3 KO

BODIPY
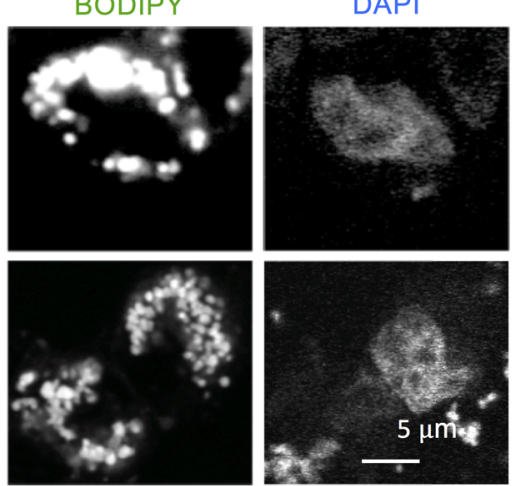

umb

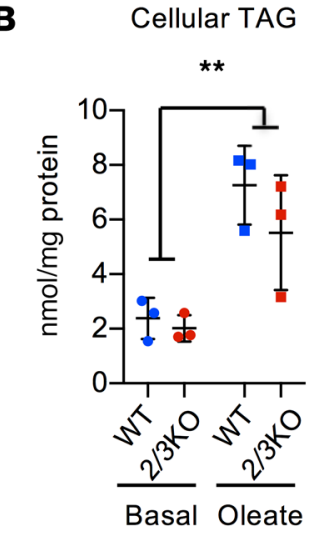

Cellular PC

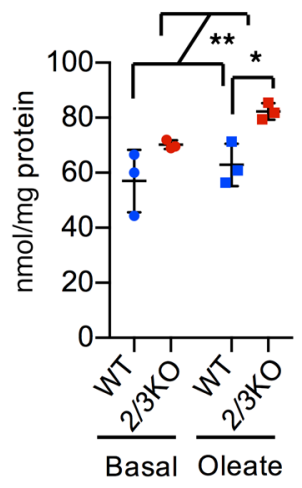

D Cellular PC levels LPIN2/3 KO

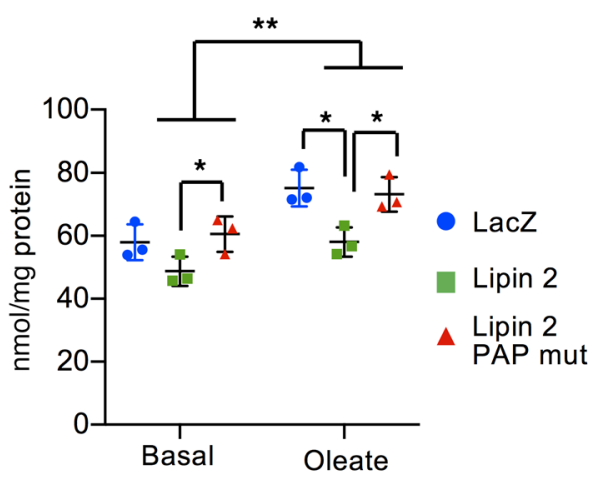

E

Cellular PA levels (oleate treated)

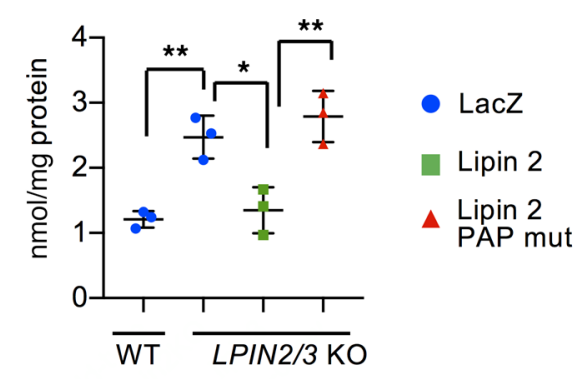

Figure 5. PAP activity from lipins 2 and 3 is required for normal lipid droplet size distribution and PC levels in enterocytes. (A) Lipins 2 and 3 are required for normal lipid droplet (LD) size and distribution. Upper: Fluorescence image of LDs in enterocyte-like HT-29 cells 24 hours after loading with $400 \mu \mathrm{M}$ oleate containing BODIPY-labeled fatty acids. Nuclei are stained with DAPI (blue). Lower: Size distribution of LDs from experiment depicted in upper panel. Diameters of LDs from at least 30 cells of each genotype were measured. (B) Lipins 2 and 3 do not influence TAG levels but do influence PC levels in HT-29 intestinal cells after acute fatty acid loading. Cellular TAG and PC were quantified under basal culture conditions and after loading with oleate for 24 hours. Average \pm $\mathrm{SD}, n=3$. ${ }^{*} P<0.05$; ${ }^{* *} P<0.01$ by $t$ test (left) or ANOVA (right). (C) Restoration of lipin 2 PAP activity to LPIN2/3-KO HT-29 cells normalizes LD size distribution. Upper: Fluorescence image of LDs in LPIN2/3-KO HT-29 cells transfected with WT lipin 2 expression vector or lipin 2 mutant lacking PAP and possessing coactivator activity. Lower: Size distribution of LDs from the experiment depicted in the upper panel. Not shown: red fluorescence derived from cotransfection with Sec61-cherry was used to identify cells successfully transfected with lipin 2, and only those cells were assessed for LD size. (D and E) Lipin 2 PAP activity restores PC and PA in LPIN2/3-KO HT-29 cells. LPIN2/3-KO cells were infected with adenovirus expressing LacZ, WT lipin 2, or PAP-mutant lipin 2. Cellular PC and PA were quantified in cells cultured in basal medium or with oleate for 24 hours. Average \pm SD, $n=3 .{ }^{*} P<0.05$; ${ }^{*} P<0.01$ by ANOVA. 
ultracentrifugation after culture under basal conditions or oleate loading. In both genotypes, lipid-containing fractions were identified by the presence of perilipin 2 (boxed lanes 1-4 in Figure 6A). In WT cells, these fractions contained the ER protein calnexin, which was more abundant in the lipid-containing fractions after oleate loading. In LPIN2/3-KO cells, however, calnexin appeared only in the high-density pellet at the bottom of the gradient. Of note, apoB48 was present in lipid-associated fractions 3-4 after oleate loading of WT cells but not LPIN2/3-KO cells (Figure 6A). ApoB48 was present in cells of both genotypes in the high-density pellet. The association of apoB48 and calnexin with lipids was restored in LPIN2/3-KO cells by complementation with WT lipin 2, but not by mutant lipin 2 harboring only coactivator activity (Figure 6B).

Lipin PAP activity influences apoB48 lipidation by modulating CCT $\alpha$. Because PC levels are elevated in lipin 2/3-deficient enterocytes - and also because those cells harbor membrane stacks - we assessed the distribution of CCT $\alpha$ in HT-29 cells grown under basal conditions and after oleate loading. CCT $\alpha$ localizes to the cytosol, nucleus, or lipid droplets. Localization to lipid droplets may activate CCT $\alpha$ enzymatic activity (43). In WT cells grown under basal conditions, CCT $\alpha$ was present in non-lipid fractions (5-10) and was associated with lipid fractions only after oleate loading. In LPIN2/3-KO cells, however, CCT $\alpha$ was associated with lipid fractions even without oleate loading (Figure 6A). In WT cells, CCT $\alpha$ protein levels increased in response to oleate loading, whereas in LPIN2/3-KO cells CCT $\alpha$ protein levels were constitutively elevated, even without oleate loading (Figure 6C). As observed with lipin 2/3-deficient mouse intestine (Figure 3F), CCT $\alpha$ mRNA levels were not elevated in human HT-29 cells (Supplemental Figure 8), suggesting that the elevated protein levels are due to posttranscriptional mechanisms. The abundance of CCT $\alpha$ protein in LPIN2/3KO cells was corrected by expression of WT lipin 2, but not by the lipin 2 PAP mutant (Figure 6C), indicating that lipin PAP activity is essential for the regulation of CCT $\alpha$ levels.

We next asked whether the increased CCT $\alpha$ activity and the resultant PC overproduction contribute to dysregulated chylomicron production in lipin 2/3-deficient enterocytes. If so, inhibition of CCT $\alpha$ activity should normalize both lipid distribution and the association of lipids with apoB48. Treatment of LPIN2/3-KO HT-29 cells with oleate in the presence of miltefosine, a CCT $\alpha$ inhibitor, reduced PC and PA levels (Figure 6D) and changed the distribution of BODIPY-labeled lipids. Rather than the small lipid droplets seen in lipin 2/3-deficient cells, we saw many larger lipid droplets and a bimodal distribution characteristic of WT cells (Figure 6E). Most notably, CCT $\alpha$ inhibition normalized the distribution of proteins, as judged by differential centrifugation. As described earlier, LPIN2/3-KO cells showed minimal occurrence of apoB48 and calnexin in the perilipin 2-containing fractions, whereas $\mathrm{CCT} \alpha$ inhibition led to a robust association of apoB48 and calnexin in those fractions (Figure 6F). In sum, our findings indicate that lipin PAP activity is a critical determinant of phospholipid synthesis, which affects TAG partitioning into the ER lumen to associate with the key chylomicron protein, apoB48.

\section{Discussion}

This study shows that lipin PAP activity in enterocytes has a critical role in chylomicron assembly - a role that is distinct from that of the
2-MAG pathway for TAG synthesis. During the postprandial phase, intestinal enterocytes absorb fatty acids and 2-MAG, which are used to synthesize TAG $(1,11)$. The TAG is incorporated into cytosolic lipid droplets for transient storage or into chylomicrons for secretion (2, 3). Here we demonstrate that lipin 2 and lipin 3 are expendable for TAG synthesis and storage in cytosolic lipid droplets but are critical for creating TAG-rich, apoB48-containing lipoproteins in the ER lumen (Figure 7). Our studies in lipin 2/3-deficient mouse intestine and HT-29 intestinal cells revealed that lipins 2 and 3 are required to maintain normal levels of CCT $\alpha$ and cellular PC homeostasis. We propose that the regulation of $\mathrm{PC}$ homeostasis by lipins 2 and 3 is a determinant of chylomicron synthesis in the ER, and defects in this process lead to an accumulation of lipid droplets in the enterocyte cytoplasm (Figure 7).

The severely impaired chylomicron production and the accumulation of cytosolic lipid droplets in Lpin2/3-KO enterocytes are reminiscent of genetic apoB deficiency. Mice that cannot synthesize apoB in the intestine have an enlarged intestine and, like Lpin2/3-KO mice, accumulate cytosolic lipid droplets (44). Human abetalipoproteinemia causes a similar lipid droplet phenotype (45). Gpat3- and Dgat1-knockout mice also accumulate intestinal lipid droplets when fed a high-fat diet $(46,47)$ but not when fed a chow diet. Although GPAT3 acts in the glycerol 3-phosphate pathway upstream of lipin PAP enzymes, GPAT3 deficiency has less severe effects than lipin 2/3 deficiency, as the rise in plasma TAG levels in GPAT3-deficient mice is blunted only by extreme lipid overload (2-4 times more oil than in the lipin 2/3 or DGAT1 studies) (47). Perhaps the less pronounced phenotype in GPAT3-deficient mice relates to compensation by GPAT4. Compared with DGAT1- or GPAT3-deficient mice, Lpin2/3-KO mice are unique in the accumulation of intestinal lipids when fed a standard chow diet, and in the accumulation of phospholipid membrane stacks in enterocytes. Lpin2/3-KO weanlings were recovered at a reduced rate compared with the expected genotype proportion. This reflects a critical role for lipin $2 / 3$ during either embryonic development or the early postnatal period, and more work will be required to establish the basis for the early lethality.

A key finding of our study is that lipins 2 and 3 are required for the association of apoB 48 with TAG during the maturation of chylomicrons in the ER. Chylomicron assembly in the ER lumen requires association of lipids with apoB 48 , and the lipidation has been proposed to occur in 2 steps: (a) the association of apoB48 with small amounts of TAG and phospholipids during its translocation into the ER and (b) the fusion of primordial apoB48containing lipid particles with apoB-free lipid droplets in the ER lumen $(48,49)$. In the liver (and presumably the intestine as well), apoB that is not lipidated during its translocation into the ER is degraded (50).

Since apoB48 accumulates in Lpin2/3-KO intestine, we propose that the first step of chylomicron assembly occurs normally, although we cannot exclude the possibility of reduced apoB degradation. However, we do not believe that the second step occurs normally. In the intestine of WT mice, BODIPY-labeled fatty acids quickly reach large lipid droplets and associate with apoB, as judged by confocal immunofluorescence microscopy. In the intestine of Lpin $2 / 3-\mathrm{KO}$ mice, however, BODIPY-labeled fatty acids accumulate in small lipid droplets that do not associate with apoB or colocalize with the 
A

Basal condition
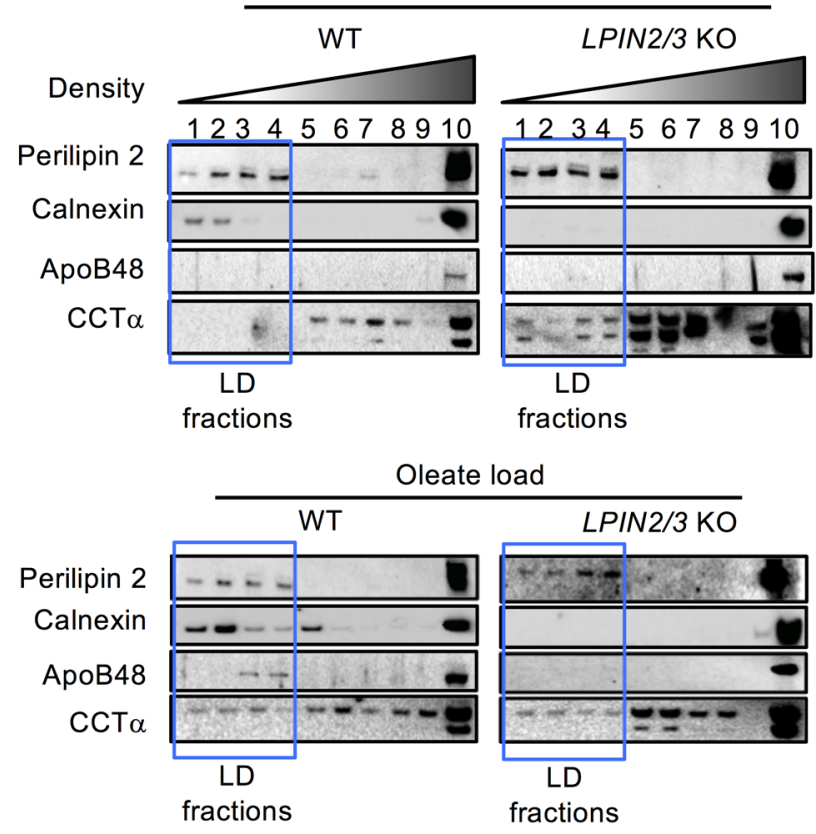

D
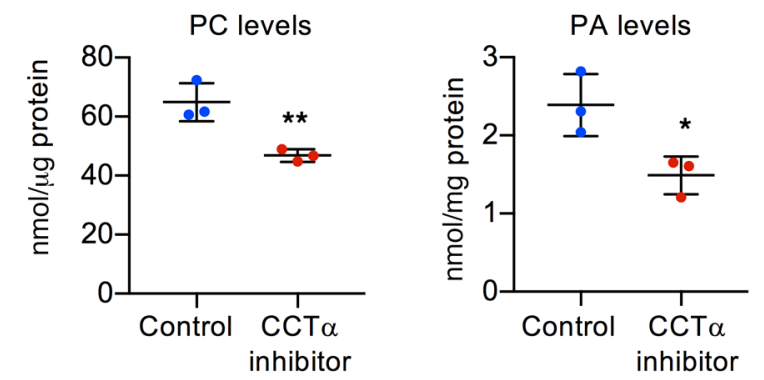

B

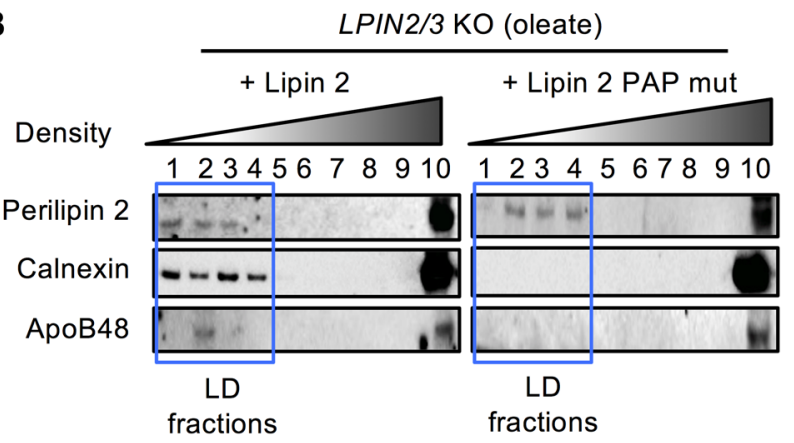

C
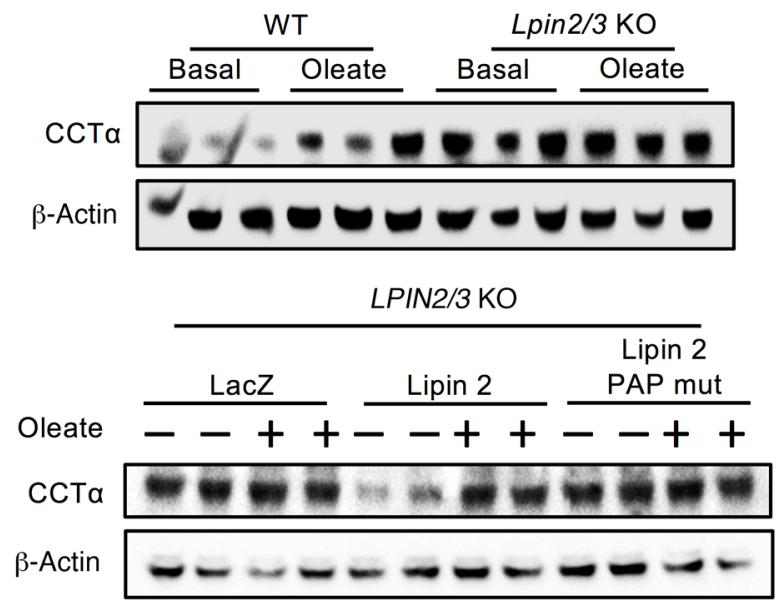

$\mathbf{F}$

Control

CCT $\alpha$ inhibitor

E

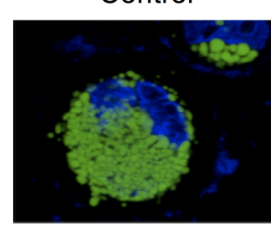

CCT $\alpha$ inhibitor
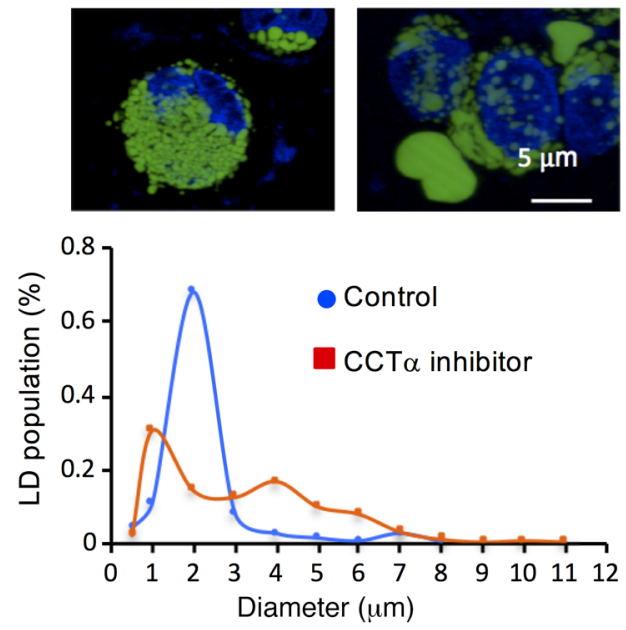

Density

Perilipin 2

Calnexin

ApoB48

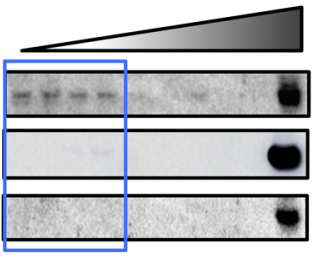

LD

fractions

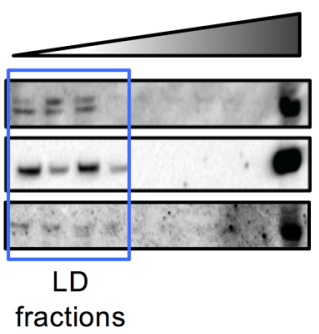


Figure 6. Lipin PAP activity is required for apoB48 association with lipid for chylomicron assembly. (A) Analysis of apoB48 association with lipids by density gradient centrifugation followed by immunoblot to detect proteins across fractions. The lipid droplet-containing (LD-containing) fractions (outlined by blue box) were defined by the presence of perilipin 2. In WT cells, lipids were associated with the ER (calnexin), and apoB48 was present in LD fractions after oleate loading. In LPIN2/3-KO cells, neither apoB48 nor calnexin was present in LD fractions, even after oleate loading. Additionally, CCT $\alpha$ levels were elevated and appeared in the LD fractions even under basal conditions, which was not observed in WT cells. (B) Lipin PAP activity is required for apoB48 association with lipids in intestinal cells. LPIN2/3-KO cells were infected with adenovirus expressing WT lipin 2 or PAP-mutant lipin 2. Cells loaded with oleate were assessed for the presence of apoB48 in LD fractions. (C) Lipin PAP activity modulates CCT $\alpha$ protein levels. Elevated CCT $\alpha$ protein levels in LPIN2/3-KO cells under basal culture conditions are normalized by introduction of adenoviral vectors for WT, but not PAP-mutant, lipin 2. (D) CCT $\alpha$ inhibitor reduces PC and PA levels in WT HT-29 cells. Average \pm SD, $n=3 .{ }^{*} P<0.05,{ }^{* *} P<0.01$ by $t$ test. (E) Fluorescence image (upper) and size distribution (lower) of LDs in LPIN2/3-KO HT-29 cells loaded with oleate without or with addition of CCT $\alpha$ inhibitor. $n \geq 30$. Nuclei are stained with DAPI (blue). (F) CCT $\alpha$ inhibitor restores calnexin and apoB48 association with lipid-containing fractions from LPIN2/3-KO cells loaded with oleate. Blots shown throughout this figure are each representative of a single experiment, but the experiments in panels $\mathbf{A}-\mathbf{C}$ and $\mathbf{F}$ are each variations of a similar experiment, such that in composite, the patterns for WT and Lpin2/3-KO mice were each replicated in at least 3 independent trials. Blots in $\mathbf{A}, \mathbf{C}$, and $\mathbf{F}$ were run with the same samples contemporaneously.

ER marker calnexin. Moreover, density gradient fractionation of extracts from human HT-29 cells after oleate loading showed that apoB48 floats in the lipid droplet (perilipin 2-positive) fractions in WT cells but not in LPIN2/3-KO cells. ApoB48 association with the lipid-containing fractions was restored by expression of lipin 2 PAP activity, but not by lipin 2 coactivator activity.

The impaired chylomicron assembly in Lpin2/3-KO enterocytes was not associated with a dearth of TAG, which likely is synthesized primarily through the 2-MAG pathway. However, LPIN2/3-KO HT-29 cells and mouse intestine accumulated PA, the substrate of lipin enzymatic activity, and PC, the major phospholipid component of chylomicrons. The accumulation of phospholipids is consistent with findings in Arabidopsis thaliana, where a deficiency of both lipin orthologs (PAH1 and PAH2) leads to ER proliferation (51), and in yeast, where loss of the single lipin ortholog leads to an expansion of the nuclear-ER membrane (52). In Lpin2/3-KO intestine, the elevated PC levels correlated with a robust increase in CCT $\alpha$ protein, which catalyzes the ratelimiting step in PC synthesis. Recent work has shown that CCT $\alpha$ is regulated at the posttranscriptional level by mTORC1 (41), and a regulator of mTORC1 is the lipid PA $(53,54)$. Consistent with this, we found that Lpin2/3 KO leads to intestinal mTORC1 activation (by assessment of phosphorylation of the downstream target p70S6 kinase). Our data suggest a relationship between lipin $2 / 3$ and CCT $\alpha$ protein levels in which loss of PAP activity leads to increased PA levels, which activate mTORC1 to increase CCT $\alpha$ protein levels and PC production.

Previous work has shown that PC regulation is critical for lipid homeostasis in the enterocyte. It has long been known that in intestinal cell lines, a threshold PC concentration is required for chylomicron synthesis (55). In vivo, de novo intestinal PC synthesis through CCT $\alpha$ is required for dietary fat absorption during high-fat feeding (56). However, little is known about the effects of increased CCT $\alpha$ and PC in enterocytes. Our data suggest that elevated PC levels contribute to impaired chylomicron biogenesis in lipin 2/3-deficient enterocytes, since normalization of PC levels with a CCT $\alpha$ inhibitor rescues defective chylomicron assembly. Altered PC composition in Lpin2/3-KO enterocytes may also impede chylomicron production. Phospholipids containing polyunsaturated acyl chains promote the transfer of TAG from the ER membrane into the ER lumen for lipoprotein production $(57,58)$. In Lpin $2 / 3-\mathrm{KO}$ intestine, the ratio of polyunsaturated PCs to total PCs was reduced. In partic- ular, $36: 4$ and $38: 4$ PCs were decreased from $22 \%$ to $16 \%$ of total PCs. This altered PC composition, together with increased total PC levels, could favor budding of TAG from the ER membrane into cytosolic lipid droplets rather than ER luminal lipid droplets for chylomicron assembly.

In summary, this study shows that lipin 2/3 PAP activity influences intestinal lipid storage and use through effects on membrane PC homeostasis and chylomicron production (Figure 7). Lipins 2 and 3 are expendable for the incorporation of dietary fatty acids into TAG, but are quite relevant to the capacity of newly synthesized TAG to enter the ER lumen for chylomicron assembly. The regulation of PA levels by lipin 2/3 may influence activation of mTORC1 to result in enhanced CCT $\alpha$ protein and PC levels. Notably, the normalization of CCT $\alpha$ levels restores chylomicron production in lipin 2/3-deficient cells. Thus, lipin PAP-mediated phospholipid homeostasis has an essential role in chylomicron synthesis that is distinct from the role of the 2-MAG pathway. The efficient processing of dietary lipids likely requires coordination between the PAP and 2-MAG pathways, such that disruption of either causes impairment in this fundamental biological process.

\section{Methods}

Mice. Lpin2-KO and Lpin3-KO mice have been described previously (34, 36), and have been bred onto a C57BL/6 background for more than 9 generations. To generate the 4 mouse genotypes used here, Lpin2 ${ }^{+/-}$

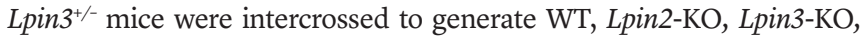
and Lpin2/3-KO mice from the same pairings. Mice were genotyped for Lpin 2 and Lpin 3 alleles as described previously $(34,36)$. Male and female cohorts were used throughout these studies; the effects of lipin $2 / 3$ deficiency did not differ in the 2 sexes. The choice of male or female cohorts for any given experiment was dictated by the availability of Lpin $2 / 3-\mathrm{KO}$ mice, of which only approximately $25 \%$ survived to adulthood. Lpin1 ${ }^{-1-}$ fld mice and WT littermates (BALB/cByJ background) were from a colony maintained at UCLA that was originally established from Lpin1 $^{+/-}$mice obtained from the Jackson Laboratory. Mice were maintained in 12-hour light/12-hour dark conditions and fed a laboratory chow diet, except for the experiments in Figure 1E, Supplemental Figure 2B, Supplemental Figure 3, and Supplemental Figure 5, where some mice were fed a highfat diet (35\% fat/33\% carbohydrate; diet F3282, BioServe) for 6 days. Whole body fat mass and lean tissue mass of live mice were determined with an EchoMRI 3-in-1 Analyzer. For food intake and fecal output determinations, mice were caged individually. After acclimation for 1 week, food intake and fecal pellet production were quantified daily for 7 days. 

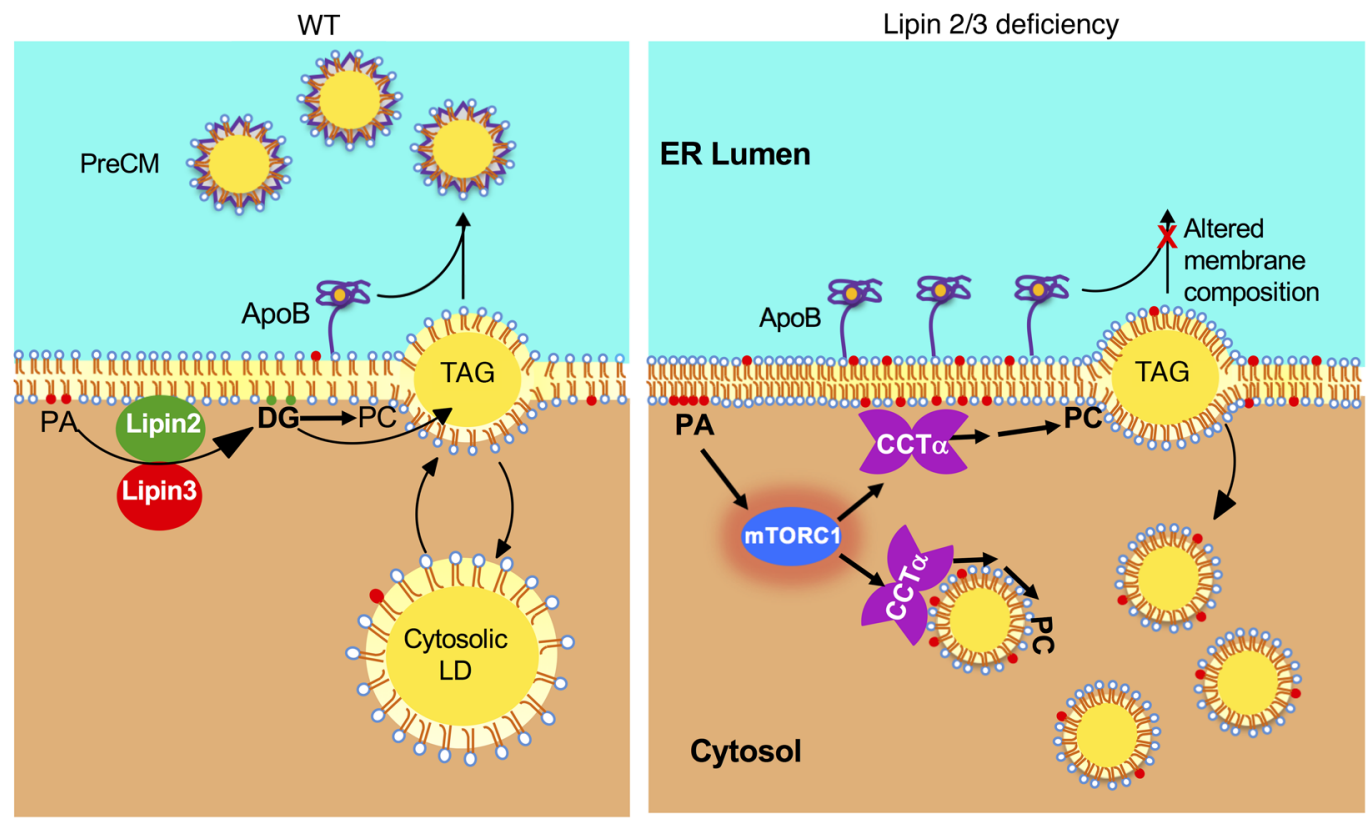
PreCM, prechylomicron
TAG, triacylglycerol
R PC, phosphatidylcholine
LD, lipid droplet
RPA, phosphatidic acid
R DG, diacylglycerol

Figure 7. Proposed role of lipins $\mathbf{2}$ and $\mathbf{3}$ in intestinal chylomicron (CM) production. Left: In WT enterocytes, TAG synthesized in the ER membrane may bud into the cytosol for storage as lipid droplets (LDs) or into the ER lumen to associate with apoB48 through the action of microsomal TAG transfer protein. The resulting prechylomicron particles (PreCMs) ultimately bud from the ER. In steps not shown, these lipoproteins subsequently mature in the Golgi and are released from enterocytes as mature CMs. Right: In the absence of lipin 2/3 PAP activity, the lipin substrate phosphatidic acid (PA) accumulates at membrane sites in the ER (and possibly additional cell membranes). Elevated PA may activate mTORC1, which enhances CCT $\alpha$ levels by increasing protein stability or translation. Increased CCT $\alpha$ contributes to elevated PC levels and altered membrane phospholipid composition and impaired PreCM formation. TAG-rich lipid droplets accumulate in the cytoplasm rather than contribute to CM formation.

Cell lines. Human intestinal colorectal carcinoma cell lines (Caco-2 [ATCC HTB-37] and HT-29 [ATCC HTB-38]) were from the American Type Culture Collection and were cultured under media conditions specified by the supplier.

Plasma analyses. Plasma total cholesterol and HDL cholesterol were quantified by the UCLA Lipid Core as described previously (59). Plasma $25(\mathrm{OH})$ vitamin D levels were determined by radioimmunoassay (Heartland Assays) (60).

Immunoblot analysis. Intestine was gently homogenized to separate the mucosa from the muscular layer in lysis buffer $(250 \mathrm{mM}$ sucrose, $20 \mathrm{mM}$ Tris, $1 \mathrm{mM}$ EDTA, 1.4\% Triton X-100, 5× Complete Mini EDTA-free Protease Inhibitor Cocktail [Roche Diagnostics], and $5 \times$ Phosphatase Inhibitor Cocktails 2 and 3 [Sigma-Aldrich]). Protein lysates were electrophoresed in 3\%-8\% acrylamide gels, transferred to nitrocellulose membranes, and incubated with primary antibodies. Primary antibodies were used at 1:1,000 dilution unless specified otherwise. Antibody against lipin 1 was a gift from Maroun Bou Kahlil and Zemin Yao (University of Ottawa, Ottawa, Canada) (61) and has been verified in Lpin1-KO mice (33). Antibody against lipin 2 was a gift from Brian Finck (Washington University, St. Louis, Missouri, USA) (62) and has been verified in Lpin2-KO mice (36). Antibody against lipin 3 (used at 1:10,000 dilution) was from Lifespan Biosciences (catalog LS-C37207) and has been verified in Lpin3-KO mice (34). Antibodies against apolipoproteins A-I,
A-IV, and B were as detailed previously (59). The polyclonal rabbit anti-mouse apoA-I and apoA-IV antibodies were used at 1:4,000 dilution, and mouse monoclonal apoB antibody was used at 1:1,000. Sources of other antibodies were as follows: $\beta$-actin (GTX109639, GeneTex), $\alpha$-tubulin 4a (GTX112141, used at 1:500, GeneTex), calnexin (ab22595, Abcam), perilipin 2/ADFP (NB110-40877, Novus Biologicals), CCT $\alpha$ (6931S, Cell Signaling Technology), p70S6 kinase (9202, Cell Signaling Technology), and phospho-p70S6 kinase, Thr 389 (9234P, Cell Signaling Technology). Speciesappropriate HRP-conjugated secondary antibodies were used to detect the primary antibody binding by enhanced chemiluminescence (GE Healthcare).

Intestinal tissue histology and immunofluorescence. For histological analysis, proximal intestine of mice fasted for 5 hours, or fasted 5 hours and refed for 2 hours was excised, fixed with $4 \%$ paraformaldehyde for 24-48 hours, embedded in Tissue-Tek paraffin wax (Sakura Finetek), sectioned by a sliding microtome REM-700 (Yamato Kohki Industrial Co., Ltd.), and stained with H\&E (33).

For analysis of fatty acid incorporation into lipids and immunostaining, proximal intestinal tissue from mice fasted for 5 hours or fasted 5 hours and gavaged with $10 \mu \mathrm{l} / \mathrm{g}$ body weight of olive oil containing BODIPY 500/510 $\mathrm{C}_{1}, \mathrm{C}_{12}$ fatty acid (D3823, $2 \mu \mathrm{g} / \mathrm{g}$; Molecular Probes) was excised, frozen in optimum cutting temperature (OCT) compound, cut into 10 - to $12-\mu \mathrm{m}$ sections, fixed for 10 
minutes with $10 \%$ neutral buffered formalin (Sigma-Aldrich), and mounted with ProLong Diamond Antifade Mountant with DAPI (ThermoFisher Scientific). Sections were examined by confocal fluorescence microscopy to assess BODIPY labeling or stained with antibodies against lipin 2, lipin 3, apoB, or calnexin (described under Immunoblot analysis section). Briefly, after 60-minute fixation with $10 \%$ neutral buffered formalin, the sections were incubated with primary antibodies (1:100-1:500) overnight at $4^{\circ} \mathrm{C}$ and examined by fluorescence microscopy with a Leica TCS-SP5 confocal laserscanning microscope equipped with an argon-krypton laser.

Electron microscopy. Mice were fasted 5 hours or were fasted 5 hours and gavaged with $100 \mu \mathrm{l}$ corn oil and allowed to digest for 2 hours, sacrificed, and perfused through the heart with PBS followed by fixative ( $2.5 \%$ glutaraldehyde in $100 \mathrm{mM}$ sodium cacodylate). The small intestine was flushed with additional fixative, and a small piece was collected and saved in fixative overnight at $4^{\circ} \mathrm{C}$. The fixed tissue was incubated with $1 \%$ osmium tetroxide for 1 hour at room temperature, washed with distilled water, and incubated with $2 \%$ uranyl acetate overnight at $4^{\circ} \mathrm{C}$ in the dark. Tissues were rinsed with distilled water and dehydrated by serial incubations with $20 \%, 30 \%, 50 \%$, $70 \%$, and $100 \%$ acetone for 30 minutes each. Samples were incubated in Spurr's resin at $70^{\circ} \mathrm{C}$ overnight. Tissue sections $(50 \mathrm{~nm})$ were prepared with a DiATOME diamond knife and a Leica Ultramicrotome, placed on 200-mesh copper grids, and stained with Reynolds lead citrate for 5 minutes. Images were captured with a 100CX JEOL electron microscope at $80 \mathrm{kV}$.

PAP activity assay. PAP activity was measured as described previously (31). Briefly, fresh proximal intestinal tissues were homogenized in lysis buffer consisting of $250 \mathrm{mM}$ sucrose, $2 \mathrm{mM}$ DTT, Phosphatase Inhibitor Cocktails 1 and 2, 1× Complete Mini EDTA-free Protease Inhibitor Cocktail, and 0.15\% Tween 20. PAP1 activity was measured in $100 \mathrm{mM}$ Tris/ $\mathrm{HCl}$ buffer, $\mathrm{pH} 7.4$, containing $5 \mathrm{mM} \mathrm{MgCl}_{2}$ and $2 \mathrm{mg} /$ $\mathrm{ml}$ fatty acid-poor bovine serum albumin with $0.6 \mathrm{mM}$ tritiated PA (approximately $6 \times 10^{4} \mathrm{dpm}$ labeled with $\left[{ }^{3} \mathrm{H}\right]$ palmitate) mixed with PA derived from egg PC, $0.4 \mathrm{mM}$ PC, $1 \mathrm{mM}$ EDTA, $1 \mathrm{mM}$ EGTA, and $200 \mu \mathrm{M}$ tetrahydrolipstatin to block the degradation of DAG. Tween 20 was adjusted to a final concentration of $0.05 \%$, and the reactions were incubated at $37^{\circ} \mathrm{C}$. Chloroform containing $0.08 \%$ olive oil was added to stop the reaction and basic alumina was added to remove any PA or $\left[{ }^{3} \mathrm{H}\right]$ palmitate formed by phospholipase A activity. The $\left[{ }^{3} \mathrm{H}\right] \mathrm{DAG}$ product was isolated and quantified by scintillation counting. Lysate protein amounts and incubation times were optimized to ensure that less than $15 \%$ of PA was consumed during incubation. Three different protein concentrations were analyzed for each sample to ensure the proportionality of the assay. Parallel analyses were done in the presence of excess $N$-ethylmaleimide $(5 \mathrm{mM})$ to assess the contribution of lipid-phosphate phosphatase activity. This latter activity was subtracted from the total activity to yield true PAP activity values. These assay conditions were chosen to maximize the PAP activity relative to that of lipid-phosphate phosphatases.

Coimmunoprecipitation. Coimmunoprecipitation studies were done to assess the interaction between lipin 2 and lipin 3. HEK293 cells were transfected with expression constructs for mouse lipin 2 with a C-terminal V5 epitope and for lipin 3 with a C-terminal Myc epitope. Cell lysates were incubated with $1 \mu \mathrm{g}$ of mouse anti-V5 antibody (Invitrogen), $4 \mu \mathrm{g}$ of mouse anti-Myc antibody (Upstate Biotechnology), or 4 $\mu \mathrm{g}$ of nonspecific mouse IgG (Jackson ImmunoResearch) overnight at $4^{\circ} \mathrm{C}$. Lysis buffer contained $1 \%$ NP-40 (Sigma-Aldrich), $1 \times$ Complete Mini Protease Inhibitor Cocktail, and 0.1\% Phosphatase Inhibitor Cocktails 2 and 3 in PBS. Antibody complexes were bound to Protein A/G PLUS-agarose beads (sc-2003, Santa Cruz Biotechnology) for 2-3 hours at $4^{\circ} \mathrm{C}$. After 3 washes with PBS plus $0.1 \%$ NP-40, protein was eluted and analyzed by Western blot. Lipin 2-V5 was detected with rabbit anti-V5 (Bethyl Laboratories; 1:5,000), and lipin 3-Myc was detected with rabbit anti-Myc (Bethyl Laboratories; 1:10,000).

Postprandial hypertriglyceridemia assessment. Mice were fasted for 5 hours (0800-1300 hours), blood was collected from a nick in the tail vein (time 0 ), and the mice were immediately gavaged with olive oil (10 $\mu \mathrm{l} / \mathrm{g}$ body weight) or oil containing BODIPY 500/510 $\mathrm{C}_{1}, \mathrm{C}_{12}$ fatty acid to assess postprandial TAG excursions and to visualize fatty acid internalization and incorporation into cellular lipids, respectively (63). Blood was collected by tail vein nick at 1, 2, 3, 4, and 5 hours after the olive oil challenge. Plasma TAG levels were measured with the Triglyceride Assay Kit (Sigma-Aldrich) as described previously (33).

Chylomicron secretion rates were assessed as described previously (64). Mice were fasted for 5 hours (0800-1300 hours), given $200 \mathrm{mg} /$ $\mathrm{kg}$ tyloxapol (Sigma-Aldrich) in $0.9 \% \mathrm{NaCl}$ by tail vein injection, and gavaged with olive oil 10 minutes later. Blood was collected as described for TAG determination.

Lipidomic analyses. Lipidomic analyses and biochemical lipid measurements were done as described previously (33, 65). Briefly, lipids were extracted from the intestinal epithelium, fecal pellets, or plasma by a modification of the Bligh and Dyer method (66). Fecal pellets were desiccated overnight before extraction. TAG, free cholesterol, cholesteryl ester, free fatty acid, phospholipid, sphingolipid, and DAG species were quantified by electrospray ionizationtandem mass spectrometry. Cellular PC, PA, and TAG levels in enterocyte-like HT-29 cells were biochemically determined with a PC assay kit (Sigma-Aldrich), total PA kit (Cell Biolabs), and serum triglyceride determination kit (Sigma-Aldrich) as described previously (33).

Generation of LPIN2/3-KO human intestinal cells with CRISPR/ Cas9. CRISPR genomic editing technology was used to delete LPIN2 and LPIN3 in HT-29 cells by established methods (67). The guide RNA sequences targeting human LPIN2 exon 2 or LPIN3 exon 2 were subcloned into the plasmid px459 (Addgene, 48319), a gift from Feng Zhang (The Broad Institute, Massachusetts Institute of Technology, Cambridge, Massachusetts, USA) (68). The 2 constructed plasmids for LPIN2 and LPIN3 were cotransfected into HT-29 cells. Two to 4 hours after transfection, transfected cells were selected for resistance to 1 $\mathrm{mg} / \mathrm{ml}$ puromycin for 72 hours, diluted, and plated as single cells/well. The resulting cell clones were screened by DNA sequencing of the target gene region and clones with suspected lipin 2/3 deficiency were confirmed by Western blot with lipin 2 and lipin 3 antibodies $(34,36)$.

Lipid droplet size distribution in HT-29 cells. WT and LPIN2/3-KO HT-29 cells were treated with $0.4 \mathrm{mM}$ oleate containing 1\% BODIPY $500 / 510 C_{1}, C_{12}$ fatty acid for 24 hours, fixed in $10 \%$ neutral buffered formalin, and imaged with a Leica TCS-SP5 confocal laserscanning microscope. Lipid droplet diameter was measured with ImageJ (http://imagej.nih.gov.ij/). For each experimental group, at least 500 droplets were analyzed.

Cell fractionation. Cells were harvested, washed with ice-cold PBS, resuspended in $2 \mathrm{ml}$ of buffer ( $250 \mathrm{mM}$ sucrose, $20 \mathrm{mM}$ Tris- $\mathrm{HCl}$ [pH 7.4], 1 mM EDTA, Roche Complete Protease Inhibitor tablet), and lysed with a tissue homogenizer. Lysates were cleared by centrifuga- 
tion at $1,000 \mathrm{~g}$ for 5 minutes and at 12,000 $g$ for 15 minutes. The supernatant was fractionated into floating lipid droplets and soluble and membrane fractions by using a 2-layer gradient (upper layer, $5 \mathrm{ml}: 50$ mM sucrose, 20 mM Tris-HCl [pH 7.4], 1 mM EDTA, Roche Complete Protease Inhibitor tablet; bottom layer, $5 \mathrm{ml}: 250 \mathrm{mM}$ sucrose, $20 \mathrm{mM}$ Tris-HCl [pH 7.4], 1 mM EDTA, Roche Complete Protease Inhibitor tablet) by centrifugation at $100,000 \mathrm{~g}$ for 12 hours. Fractions were collected, and the protein concentration of each fraction was determined with the Pierce BCA protein assay kit (ThermoFisher Scientific). Fractions were analyzed by immunoblot, as described above.

Gene expression analysis. RNA was extracted with TRIzol (Invitrogen). cDNA was synthesized and analyzed by quantitative PCR (qPCR) as described previously (33). The qPCR primer sequences are given below; all are mouse unless otherwise indicated. Acox1 CAGGAAGAGCAAGGAAGTGG, CCTTTCTGGCTGATCCCATA; ASBT ATGCAGAACA CGCAGCTATG, GCTCCGTTCCATTTTCTTTG; Аров CAgTATTCTGCCACTGCAACC, AgGACTTCACTAGATAAGGTCC; CD36, GAGACTGGGACCATTGGTGA, TATATGTAGGC TCATCCACTAC; Pcyt1 GGGCCAGTGACAGCTAGGGAAA, CGAGGTCAGAGTCCCTCGCATT; PCYT1A (human) CGGCAACCAGCTCCTTTTTCTG, CGGGCGTGACCAGAGTGAAATA; Cpt1a AAACCCACCAGGCTACAGTG， TCCTTGTAATGTGCGAGCTG; Dgat1 TGCTACGACGAGTTCTTGAG， CTCTGCCACAGCATTGAGAC; Dgat2 GGCGCTACTTCCGAGACTAC, TGGTCAGCAGGTTGTGTGTC; Fabp1 GTCAAGGCAGTCGTCAAGCT, TCTTGTAGACAATGTCGCCCA; Fabp2 TAGACCGGAACGAGAACTATG, AATGGTCCAGGCCCCAGTGA; LPIN2 (human) CCTCTCCTCAGACCAGATCG, GGAGAATCTGTCCCAAAGCA; LPIN3 (human) CACTCCACCCTCCACTCCTA, ACAGGTAGATGGTGGCCTTG; Mogat2 TGGTGGTACCTGGACTGGGACA, GTGGAAGCCCGCGATGTAGTtC; $M t t p$, AGCTGCAATCTGGACTAAAGG, ACTGTGACGTCGCTGGTTATC; Pnpla2 GCCAACGCCACTCACATCTACG, GGgTCTTtAGTAGACAGCCACG; Sar1b CAG-
CACGTCCCAACGCTACATC， ACGTGCCCACCCAGATCAAAA; $36 b 4$ AGTACACCTTCCCACTTACTG, ACCAAGTCAAGAGACTGTCTC; Tbp ACCCTTCACCAATGACTCCTATG, ATGATGACTGCAGCAAATCGC.

Statistics. The observed birth rate of Lpin2/3-KO mice was compared with the expected number (according to Mendelian inheritance) by $\chi^{2}$ test. Quantitative results are presented as mean \pm SD. Two-way ANOVA or 1-way ANOVA, followed by Bonferroni's correction was used for multiple comparisons (Stata 11). A value of $P<0.05$ was considered statistically significant.

Study approval. The Institutional Animal Care and Use Committee of UCLA approved all animal experimental protocols.

\section{Author contributions}

PZ, LSC, ER, LJB, JYL, AG, JRD, and DNB performed experiments. PZ, LSC, LGF, PT, SGY, and KR designed experiments. KR, $\mathrm{PZ}$, and LSC wrote the manuscript.

\section{Acknowledgments}

We thank Christopher N. Goulbourne for performing electron microscopy. This work was supported by NIH/National Heart, Lung, and Blood Institute grants P01 HL090553 and P01 HL028481.

Address correspondence to: Karen Reue, Human Genetics, Gonda 6357B, David Geffen School of Medicine at UCLA, 695 Charles E. Young Drive South, Los Angeles, California 90095, USA. Phone: 310.794.5631; Email: reuek@ucla.edu.

JYL's present address is: Harvard School of Public Health, Boston, Massachusetts, USA.

JRD's present address is: The Jackson Laboratory, Bar Harbor, Maine, USA.
1. D’Aquila T, Hung YH, Carreiro A, Buhman KK. Recent discoveries on absorption of dietary fat: Presence, synthesis, and metabolism of cytoplasmic lipid droplets within enterocytes. Biochim Biophys Acta. 2016;1861(8 Pt A):730-747.

2. Abumrad NA, Davidson NO. Role of the gut in lipid homeostasis. Physiol Rev. 2012;92(3):1061-1085.

3. Sturley SL, Hussain MM. Lipid droplet formation on opposing sides of the endoplasmic reticulum. J Lipid Res. 2012;53(9):1800-1810.

4. Mansbach CM, Siddiqi S. Control of chylomicron export from the intestine. Am J Physiol Gastrointest Liver Physiol. 2016;310(9):G659-G668.

5. Pan X, Hussain MM. Gut triglyceride production. Biochim Biophys Acta. 2012;1821(5):727-735.

6. Kindel T, Lee DM, Tso P. The mechanism of the formation and secretion of chylomicrons. Atheroscler Suppl. 2010;11(1):11-16.

7. Wang H, Airola MV, Reue K. How lipid droplets “TAG" along: Glycerolipid synthetic enzymes and lipid storage. Biochim Biophys Acta Mol Cell Biol Lipids. 2017;1862(10 Pt B):1131-1145.

8. Coleman RA, Mashek DG. Mammalian triacylglycerol metabolism: synthesis, lipolysis, and signaling. Chem Rev. 2011;111(10):6359-6386.

9. Zhang P, Reue K. Lipin proteins and glycerolipid metabolism: Roles at the ER membrane and beyond. Biochim Biophys Acta Biomembr. 2017;1859(9 Pt B):1583-1595.

10. Reue K, Brindley DN. Thematic review series: Glycerolipids. Multiple roles for lipins/phosphatidate phosphatase enzymes in lipid metabolism. JLipid Res. 2008;49(12):2493-2503.

11. Yen CL, Nelson DW, Yen MI. Intestinal triacylglycerol synthesis in fat absorption and systemic energy metabolism. JLipid Res. 2015;56(3):489-501.

12. Yen CL, et al. Deficiency of the intestinal enzyme acyl CoA:monoacylglycerol acyltransferase-2 protects mice from metabolic disorders induced by high-fat feeding. Nat Med. 2009;15(4):442-446.

13. Johnston JM, Rao GA, Lowe PA, Schwarz BE. The nature of the stimulatory role of the supernatant fraction on triglyceride synthesis by the alpha-glycerophosphate pathway. Lipids. 1967;2(1):14-20.

14. Smith ME, Sedgwick B, Brindley DN, Hübscer G. The role of phosphatidate phosphohydrolase in glyceride biosynthesis. Eur J Biochem. 1967;3(1):70-77.

15. Hübscher G, Brindley DN, Smith ME, Sedgwick B. Stimulation of biosynthesis of glyceride. Nature. 1967;216(5114):449-453.

16. Short VJ, Dils R, Brindley DN. Enzymes of glycerolipid synthesis in small-intestinal mucosa of foetal and neonatal guinea pigs. Biochem J. 1975;152(3):675-679.

17. Hamilton RL, Wong JS, Cham CM, Nielsen LB, Young SG. Chylomicron-sized lipid particles are formed in the setting of apolipoprotein B deficiency. J Lipid Res. 1998;39(8):1543-1557.

18. Hussain MM, Nijstad N, Franceschini L. Regulation of microsomal triglyceride transfer protein. Clin Lipidol. 2011;6(3):293-303.

19. Raabe M, et al. Analysis of the role of microsomal triglyceride transfer protein in the liver of tissue-specific knockout mice. J Clin Invest. 1999;103(9):1287-1298.

20. Dash S, Xiao C, Morgantini C, Lewis GF. New insights into the regulation of chylomicron production. Annu Rev Nutr. 2015;35:265-294.

21. Phan CT, Tso P. Intestinal lipid absorption and transport. Front Biosci. 2001;6:D299-D319.

22. Shi Y, Cheng D. Beyond triglyceride synthesis: the dynamic functional roles of MGAT and DGAT enzymes in energy metabolism. Am J Physiol Endocrinol Metab. 2009;297(1):E10-E18.

23. Luchoomun J, Hussain MM. Assembly and secretion of chylomicrons by differentiated Caco-2 cells. Nascent triglycerides and preformed phospholipids are preferentially used for lipoprotein assembly. J Biol Chem. 1999;274(28):19565-19572. 
24. Csaki LS, Dwyer JR, Fong LG, Tontonoz P, Young SG, Reue K. Lipins, lipinopathies, and the modulation of cellular lipid storage and signaling. Prog Lipid Res. 2013;52(3):305-316.

25. Finck BN, et al. Lipin 1 is an inducible amplifier of the hepatic PGC-1alpha/PPARalpha regulatory pathway. Cell Metab. 2006;4(3):199-210.

26. Kim HB, et al. Lipin 1 represses NFATc4 transcriptional activity in adipocytes to inhibit secretion of inflammatory factors. Mol Cell Biol. 2010;30(12):3126-3139.

27. Chen Z, Gropler MC, Mitra MS, Finck BN. Complex interplay between the lipin 1 and the hepatocyte nuclear factor $4 \alpha(\mathrm{HNF} 4 \alpha)$ pathways to regulate liver lipid metabolism. PLOS ONE. 2012;7(12):e51320.

28. Koh YK, et al. Lipin1 is a key factor for the maturation and maintenance of adipocytes in the regulatory network with CCAAT/enhancer-binding protein alpha and peroxisome proliferatoractivated receptor gamma 2.J Biol Chem. 2008;283(50):34896-34906.

29. Peterson TR, et al. mTOR complex 1 regulates lipin 1 localization to control the SREBP pathway. Cell. 2011;146(3):408-420.

30. Donkor J, et al. A conserved serine residue is required for the phosphatidate phosphatase activity but not the transcriptional coactivator functions of lipin-1 and lipin-2. J Biol Chem. 2009;284(43):29968-29978.

31. Donkor J, Sariahmetoglu M, Dewald J, Brindley DN, Reue K. Three mammalian lipins act as phosphatidate phosphatases with distinct tissue expression patterns. J Biol Chem. 2007;282(6):3450-3457.

32. Zeharia A, et al. Mutations in LPIN1 cause recurrent acute myoglobinuria in childhood. Am J Hum Genet. 2008;83(4):489-494.

33. Zhang P, Verity MA, Reue K. Lipin-1 regulates autophagy clearance and intersects with statin drug effects in skeletal muscle. Cell Metab. 2014;20(2):267-279.

34. Csaki LS, et al. Lipin-1 and lipin-3 together determine adiposity in vivo. Mol Metab. 2014;3(2):145-154

35. Langner CA, et al. The fatty liver dystrophy (fld) mutation. A new mutant mouse with a developmental abnormality in triglyceride metabolism and associated tissue-specific defects in lipoprotein lipase and hepatic lipase activities. J Biol Chem. 1989;264(14):7994-8003.

36. Dwyer JR, et al. Mouse lipin-1 and lipin-2 cooperate to maintain glycerolipid homeostasis in liver and aging cerebellum. Proc Natl Acad Sci USA. 2012;109(37):E2486-E2495.

37. Lordén G, et al. Lipin-2 regulates NLRP3 inflammasome by affecting $\mathrm{P} 2 \mathrm{X} 7$ receptor activation. JExp Med. 2017;214(2):511-528.

38. Ferguson PJ, et al. Homozygous mutations in
LPIN2 are responsible for the syndrome of chronic recurrent multifocal osteomyelitis and congenital dyserythropoietic anaemia (Majeed syndrome). JMed Genet. 2005;42(7):551-557.

39. Vergnes L, Lee JM, Chin RG, Auwerx J, Reue K. Diet1 functions in the FGF15/19 enterohepatic signaling axis to modulate bile acid and lipid levels. Cell Metab. 2013;17(6):916-928.

40. Liu GH, et al. Lipin proteins form homo- and hetero-oligomers. Biochem J. 2010;432(1):65-76.

41. Quinn WJ, et al. mTORC1 stimulates phosphatidylcholine synthesis to promote triglyceride secretion. J Clin Invest. 2017;127(11):4207-4215.

42. Rehnmark S, Giometti CS, Slavin BG, Doolittle $\mathrm{MH}$, Reue K. The fatty liver dystrophy mutant mouse: microvesicular steatosis associated with altered expression levels of peroxisome proliferator-regulated proteins. J Lipid Res. 1998;39(11):2209-2217.

43. Krahmer N, et al. Phosphatidylcholine synthesis for lipid droplet expansion is mediated by localized activation of CTP:phosphocholine cytidylyltransferase. Cell Metab. 2011;14(4):504-515.

44. Young SG, et al. A genetic model for absent chylomicron formation: mice producing apolipoprotein $\mathrm{B}$ in the liver, but not in the intestine. J Clin Invest. 1995;96(6):2932-2946.

45. Bouma ME, et al. Description of two different patients with abetalipoproteinemia: synthesis of a normal-sized apolipoprotein B-48 in intestinal organ culture. J Lipid Res. 1990;31(1):1-15.

46. Buhman KK, et al. DGAT1 is not essential for intestinal triacylglycerol absorption or chylomicron synthesis. J Biol Chem. 2002;277(28):25474-25479.

47. Khatun I, et al. Characterization of a novel intestinal glycerol-3-phosphate acyltransferase pathway and its role in lipid homeostasis. J Biol Chem. 2016;291(6):2602-2615.

48. Fisher EA, Ginsberg HN. Complexity in the secretory pathway: the assembly and secretion of apolipoprotein B-containing lipoproteins. J Biol Chem. 2002;277(20):17377-17380.

49. Brodsky JL, Fisher EA. The many intersecting pathways underlying apolipoprotein B secretion and degradation. Trends Endocrinol Metab. 2008;19(7):254-259.

50. Fisher EA, et al. The degradation of apolipoprotein B100 is mediated by the ubiquitin-proteasome pathway and involves heat shock protein 70. J Biol Chem. 1997;272(33):20427-20434.

51. Eastmond PJ, Quettier AL, Kroon JT, Craddock C, Adams N, Slabas AR. Phosphatidic acid phosphohydrolase 1 and 2 regulate phospholipid synthesis at the endoplasmic reticulum in Arabidopsis. Plant Cell. 2010;22(8):2796-2811.

52. Santos-Rosa H, Leung J, Grimsey N, Peak-Chew $\mathrm{S}$, Siniossoglou S. The yeast lipin Smp 2 couples phospholipid biosynthesis to nuclear membrane growth. EMBO J. 2005;24(11):1931-1941.
53. Fang Y, Vilella-Bach M, Bachmann R, Flanigan A, Chen J. Phosphatidic acid-mediated mitogenic activation of mTOR signaling. Science. 2001;294(5548):1942-1945.

54. Menon D, et al. Lipid sensing by mTOR complexes via de novo synthesis of phosphatidic acid. J Biol Chem. 2017;292(15):6303-6311.

55. Field FJ, Born E, Chen H, Murthy S, Mathur $\mathrm{SN}$. Regulation of apolipoprotein B secretion by biliary lipids in CaCo-2 cells. JLipid Res. 1994;35(5):749-762.

56. Kennelly JP, et al. Intestinal de novo phosphatidylcholine synthesis is required for dietary lipid absorption and metabolic homeostasis J Lipid Res. 2018;59(9):1695-1708.

57. Hashidate-Yoshida T, et al. Fatty acid remodeling by LPCAT3 enriches arachidonate in phospholipid membranes and regulates triglyceride transport. Elife. 2015;4: e06328.

58. Rong X, et al. Lpcat3-dependent production of arachidonoyl phospholipids is a key determinant of triglyceride secretion. Elife. 2015;4:e06557.

59. Link JC, et al. Increased high-density lipoprotein cholesterol levels in mice with $\mathrm{XX}$ versus $\mathrm{XY}$ sex chromosomes. Arterioscler Thromb Vasc Biol. 2015;35(8):1778-1786.

60. Hollis BW, Kamerud JQ, Selvaag SR, Lorenz JD, Napoli JL. Determination of vitamin D status by radioimmunoassay with an $125 \mathrm{I}$-labeled tracer. Clin Chem. 1993;39(3):529-533.

61. Manmontri B, et al. Glucocorticoids and cyclic AMP selectively increase hepatic lipin-1 expression, and insulin acts antagonistically. J Lipid Res. 2008;49(5):1056-1067.

62. Gropler MC, et al. Lipin 2 is a liver-enriched phosphatidate phosphohydrolase enzyme that is dynamically regulated by fasting and obesity in mice. J Biol Chem. 2009;284(11):6763-6772.

63. Wang B, et al. Intestinal phospholipid remodeling is required for dietary-lipid uptake and survival on a high-fat diet. Cell Metab. 2016;23(3):492-504.

64. Masuda D, et al. Chylomicron remnants are increased in the postprandial state in CD36 deficiency. J Lipid Res. 2009;50(5):999-1011.

65. Zhang P, Takeuchi K, Csaki LS, Reue K. Lipin-1 phosphatidic phosphatase activity modulates phosphatidate levels to promote peroxisome proliferator-activated receptor $\gamma$ (PPAR $\gamma$ ) gene expression during adipogenesis. J Biol Chem . 2012;287(5):3485-3494.

66. Bligh EG, Dyer WJ. A rapid method of total lipid extraction and purification. Can J Biochem Physiol.1959;37(8):911-917.

67. Cong L, et al. Multiplex genome engineering using CRISPR/Cas systems. Science. 2013;339(6121):819-823.

68. Ran FA, Hsu PD, Wright J, Agarwala V, Scott DA, Zhang F. Genome engineering using the CRISPRCas9 system. Nat Protoc. 2013;8(11):2281-2308. 